\title{
A CMOS IC-based multisite measuring system for stimulation and recording in neural preparations in vitro
}

\author{
Takashi Tateno ${ }^{1,2 *}$ and Jun Nishikawa ${ }^{2}$ \\ Special Research Promotion Group, Graduate School of Frontier Biosciences, Osaka University, Osaka, Japan \\ ${ }^{2}$ Bioengineering and Bioinformatics, Graduate School of Information Science and Technology, Hokkaido University, Sapporo, Japan
}

\section{Edited by:}

Michele Giugliano, University of

Antwerp, Belgium

Reviewed by:

Valentina Pasquale, Fondazione Istituto Italiano di Tecnologia, Italy

Max Eickenscheidt, Max Planck

Society, Germany

*Correspondence:

Takashi Tateno, Bioengineering and Bioinformatics, Graduate School of Information Science and Technology, Hokkaido University, Kita 14, Nishi 9 , Kita-ku, Sapporo 060-0814, Japan e-mail: tateno@ist.hokudai.ac.jp
In this report, we describe the system integration of a complementary metal oxide semiconductor (CMOS) integrated circuit (IC) chip, capable of both stimulation and recording of neurons or neural tissues, to investigate electrical signal propagation within cellular networks in vitro. The overall system consisted of three major subunits: a $5.0 \times 5.0 \mathrm{~mm}$ CMOS IC chip, a reconfigurable logic device (field-programmable gate array, FPGA), and a PC. To test the system, microelectrode arrays (MEAs) were used to extracellularly measure the activity of cultured rat cortical neurons and mouse cortical slices. The MEA had 64 bidirectional (stimulation and recording) electrodes. In addition, the CMOS IC chip was equipped with dedicated analog filters, amplification stages, and a stimulation buffer. Signals from the electrodes were sampled at $15.6 \mathrm{kHz}$ with 16 -bit resolution. The measured input-referred circuitry noise was $10.1 \mu \mathrm{V}$ root mean square $(10 \mathrm{~Hz}$ to $100 \mathrm{kHz})$, which allowed reliable detection of neural signals ranging from several millivolts down to approximately $33 \mu V_{p p}$. Experiments were performed involving the stimulation of neurons with several spatiotemporal patterns and the recording of the triggered activity. An advantage over current MEAs, as demonstrated by our experiments, includes the ability to stimulate (voltage stimulation, 5-bit resolution) spatiotemporal patterns in arbitrary subsets of electrodes. Furthermore, the fast stimulation reset mechanism allowed us to record neuronal signals from a stimulating electrode around $3 \mathrm{~ms}$ after stimulation. We demonstrate that the system can be directly applied to, for example, auditory neural prostheses in conjunction with an acoustic sensor and a sound processing system.

Keywords: bidirectional electrode, CMOS IC technology, low-noise amplifier, neural networks, noise suppressor

\section{INTRODUCTION}

Multielectrode arrays (MEAs) allow researchers to stimulate and record multiple electrical signals at the single-cell level, and thus have become an indispensable tool in the study of neural network properties such as network formation (Maeda et al., 1995; Kamioka et al., 1996), network dynamics (Streit et al., 2001; Jimbo et al., 2003), and signal processing (Meister et al., 1994). An advantage of MEAs is that they can be arranged in planar structures using microfabrication technology, allowing for the investigation not only of neuronal cultures and brain slices in vitro, but also of animal tissues in vivo (Rubehn et al., 2009; Hampson et al., 2012; Hottowy et al., 2012). Several recent studies also reported the system integration of a complementary metal oxide semiconductor (CMOS) integrated circuit (IC) chip that is capable of both recording and stimulation of neurons or neural tissues (Hafizovic et al., 2007; Hottowy et al., 2008).

In particular, recent studies have reported the development of prototype bidirectional (recording and stimulation) systems in vitro and in vivo (Madhavan et al., 2006; Newman et al., 2012). However, in contrast to both modern MEA-based systems that are capable of recording complex spatiotemporal neural network activity patterns and to recent closed-loop electrophysiological systems (Rolston et al., 2010), the functionality of these CMOS IC-based systems with respect to the electrical stimulation of complex neuronal activity is very limited. The stimulation is usually restricted to a single electrode or several electrodes following the same stimulation protocol (Dabrowski et al., 2004). More sophisticated approaches include the definition of several independent stimulation signals that can be repeated by groups of electrodes (Charvet et al., 2010; Frey et al., 2010) or the fast switching of single-channel stimulation circuitry between electrodes (Wagenaar et al., 2004). In addition, neural stimulation is a key function performed by recent implantable medical devices for applications requiring many stimulation sites such as retinal prostheses for the blind and cochlear implants for the hearing disability. Such medical devices for the sensory neural prostheses need a completely flexible spatiotemporal stimulation to encode sensory information in the environment. However, although these CMOS IC-based systems can generate patterns of stimulation signals that are distributed in space and time, few of them exploit the potential of modern MEAs to elicit complex, arbitrarily defined activity patterns in a large number of neurons (Hafizovic et al., 2007).

An additional problem common to all systems aiming at simultaneous electrical stimulation and recording is stimulation 
artifacts. The electrical signals applied to activate neurons are sensed by all electrodes of the array as stimulus-related artifacts, with amplitudes several orders of magnitude larger than the amplitudes of the recorded action potentials. This can result in the saturation of the recording amplifier and makes the detection of the neuron response very difficult. Although the artifacts can be vastly reduced in magnitude by the optimization of the stimulation circuitry and experimental protocol (Jimbo et al., 2003; Brown et al., 2008; Frey et al., 2010), as well as by additional signal post-processing during data analysis (Wichmann, 2000; Wagenaar and Potter, 2002; Gnadt et al., 2003; Sekirnjak et al., 2006), most current CMOS IC-based MEA systems are unable to record neuronal responses on the stimulating electrode for at least $2 \mathrm{~ms}$ following the stimulus, and for at least half a millisecond on the nearby non-stimulating electrodes. In comparison, the delay of the elicited action potential in response to the stimulation pulse can be as short as $100 \mu$ s (Sekirnjak et al., 2008) and the duration of the recorded pulse is on the order of a millisecond. Therefore, the detection and proper identification of fast neuronal responses with such systems are extremely difficult. To avoid large stimulation artifacts and to archive charge balancing, some design techniques for neural stimulators have been presented (Hafizovic et al., 2007; Rolston et al., 2009). However, there are few reports regarding application of CMOS IC-based MEA systems to brain slice preparation (David-Pur et al., 2014), although some studies have described possible applications in the future (Hutzler et al., 2006; Berdondini et al., 2009).

In this study, we report on the system integration of a CMOS IC chip that is capable of both stimulation and recording of neurons or neural tissues, permitting investigation of electrical signal propagation within cellular networks in vitro. In our system, the frequency range of recording signals is adjustable for specific targets, including action potentials and local field potentials (FPs). In addition, although stimulation in typical CMOS IC-based MEA systems has been strictly limited to simple pulse patterns and few electrodes, we describe an application-specific integrated circuit (ASIC) system designed for multisite electrical stimulation of neural tissue using standard MEAs. Our design is intended for applications in systems requiring simultaneous stimulation and recording of signals from various types of neural tissue, both in vitro (especially in brain slice preparations) and in vivo. The developed ASIC comprises 64 independent stimulation channels, any subset of which may be stimulated, and can generate almost arbitrarily defined mono/bi-polar voltage pulses below an amplitude of 2.5 V with 5-bit resolution. Each channel is also equipped with a stimulation artifact suppressor controlled in real time, which reduces the dead time of the system after each stimulation pulse. The system we developed can be used for conventional electrophysiological experiments in vitro, and the technology is also a foundation for future auditory neural prostheses applied to the auditory central nervous system.

\section{MATERIALS AND METHODS TOTAL SYSTEM DESCRIPTION: RECORDING AND STIMULATION}

The total system, excluding cellular and/or tissue interfaces, includes three components: (1) the CMOS ASIC chip, (2) the FPGA board for the chip evaluation, and (3) the PC (Figure 1).
In the ASIC chip, each of the 64 readout channels includes amplification and filter stages. The gain and filter settings of the stages can be programed digitally. Sixteen channels are then multiplexed and buffered by each of four amplifiers in the next stage, followed by analog-to-digital converter (ADC) processing at $15.6 \mathrm{kHz}$ per channel, providing 16-bit resolution in the FPGA board. The data are subsequently transferred to a data processing unit in the FPGA. In contrast, two digital interfaces (DIs) in the chip decode commands that are used to define parameters of the stimulation and operation control (DI1) as well as those of signal amplitude and bias settings (DI2). To feed a voltage pulse series into electrodes, the system includes 64 sets of stimulation buffers and 5-bit digital-to-analog converters (DACs). The command decoder in the DI1 allows for specification of which DAC to use and configures the buffer in voltage mode.

\section{SIGNAL PROCESSING CIRCUITRY}

The signal processing circuitry was designed to meet the specification of extracellular (capacitive) recordings of neural cell activity. Neural signals usually have amplitudes of hundreds of microvolts and frequency ranges from approximately $100 \mathrm{~Hz}$ to $6 \mathrm{kHz}$ (Najafi, 1994). Many CMOS IC-based neural amplifier designs have been reported in the literature (Harrison and Charles, 2003; Mohseni and Najafi, 2004; Olsson et al., 2005; Perelman and Ginosar, 2007; for review, Gosselin, 2011). Most amplifiers achieve a low input-referred noise of $5 \mu \mathrm{V}$ for bandwidths of 5-10 kHz. In particular, the use of a CMOS-bipolar pseudoresistor element as a high-resistance element and on-chip DCcoupling capacitors enables the amplifier to reject large DC offsets at electrode-tissue interfaces (Harrison and Charles, 2003) while allowing neural signals of interest to pass. Since the technique permits high-resistance elements to be implemented in a small area on-chip, large off-chip components are not needed. The amplifier reported by Harrison and Charles (2003) uses a standard wideoutput swing operational transconductance amplifier (OTA) with capacitive feedback to realize a gain of approximately $40 \mathrm{~dB}$, and presents design techniques that minimize the input-referred noise of the amplifier by operating some devices of the OTA in strong inversion to minimize their noise contributions. Although our amplifiers in this study use a similar technique, they can be configured for either recording neural spikes or local FPs by altering their bandwidth. Particularly, in usual extracellular recordings, offset and drift that occur at the electrode can be significantly larger than the signal amplitude. These effects need to be eliminated to permit amplification of the small neural signals. To this end, higher and lower corner frequencies were respectively used in low- and high-pass filters. The high-pass filter (HPF) has been filter with one of several adjustable corner frequencies (e.g., 0.10, $1.0,10$, or $100 \mathrm{~Hz}$ ). Next, the signals pass the low-pass filter (LPF) with one of several tunable (reconfigurable) corner frequencies (e.g., 1.0, 2.5, 5.0, 7.5, and $10 \mathrm{kHz}$ ) to prevent high-frequency aliasing and to limit noise bandwidth. Finally, a buffer with larger bandwidth has been realized to further amplify the signal and to allow multiplexing (cf. Figure 1).

In this study, we used a conventional, variable-bandwidth lownoise amplifier (LNA) for the neural recording system (Zou et al., 2009), as shown in Figure 2A. The LNA consists of an OTA, 


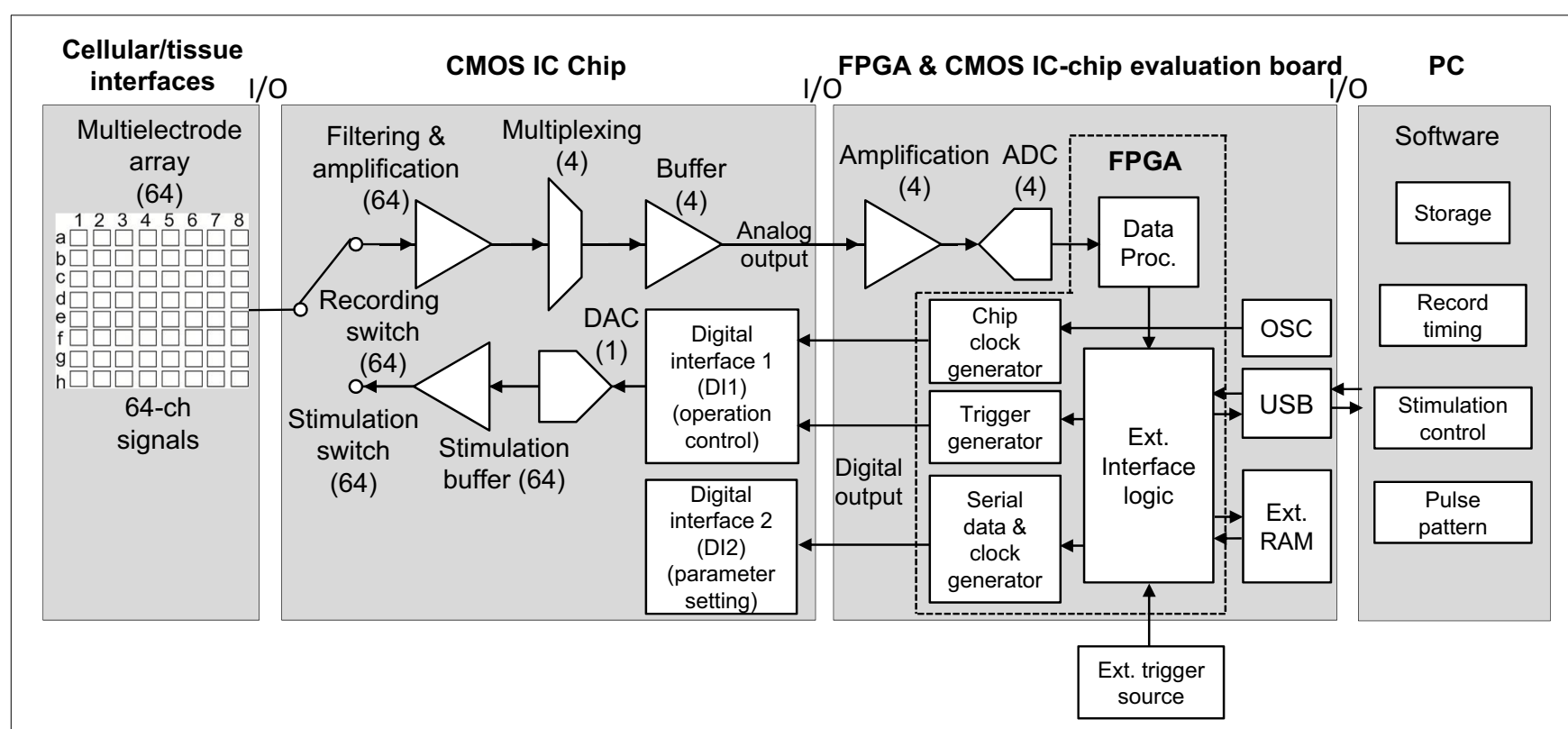

FIGURE 1 | System overview with respect to signal and data flow. In this system, neural signals and recorded data are processed from left to right (cellular/tissue interfaces to a PC), while stimulation data propagate from right to left (a PC to cellular/tissue interfaces). In this study, the leftmost block labeled "Cellular/tissue interfaces" is specifically a planar MEA, but other types of multielectrode (e.g., penetrate electrode or surface electrode) array can be used. The second block labeled "CMOS IC chip" includes components in multiple realizations. In all blocks, numbers in brackets indicate the number of realizations per chip; e.g., 64 electrodes, 64 amplifiers, 4 buffers, and so on. The arrows indicate the direction of signals and data flow. In the FPGA and CMOS IC evaluation board block, "OSC" represents an oscillator. For the FPGA and CMOS IC evaluation board and PC software, see text and Figures 3, 4.
AC-coupled input capacitors $C_{1}$, feedback capacitors $C_{2}$, and tunable pseudoresistors as feedback resistors $R_{\mathrm{f}}$. The input capacitors in the circuit that are not directly used are represented as $C_{\mathrm{g}}$. The corner frequency of the HPF is controlled by the tunable pseudoresistors. The filter cancels drift and offset of the electrode, which can be significantly larger than the signal amplitude. The mid-band voltage gain is set by $C_{1} / C_{2}$, and the lower corner frequency is adjusted by the bandwidth of the OTA and is given by the capacitor $C_{2}$ and resistor $R_{\mathrm{f}}$. The value of $C_{2}$ is a trade-off between gain accuracy and corner frequency. To realize a corner frequency of $1 \mathrm{~Hz}$, for example, a large resistance $R_{\mathrm{f}}$ is required (e.g., $R_{\mathrm{f}}=3.2 \mathrm{~T} \Omega$ ). Such large resistance has been realized by using a MOS resistor (Harrison and Charles, 2003). The lower corner frequency can be tuned from approximately $1 \mathrm{~Hz}$ to about $1 \mathrm{kHz}$, which corresponds to a resistor with resistances between $32 \mathrm{G}$ and $32 \mathrm{~T} \Omega$ (Table 1). In this study, the LNA consisted of a fully differential operational amplifier, a buffer amplifier, AC-coupled capacitors $C_{1 i}(i=1, \ldots, 4)$, feedback capacitors $C_{2}$ and $C_{3 i}(i=1, \ldots, 5)$, gain and corner-frequency selector switches, and feedback resistors implemented by cascade MOSFETs (Figure 2B). The corner frequency of the LPF was controlled by the switches of the capacitors $C_{3 i}(i=2, \ldots, 5)$. Thus, the LNA realizes high-input impedance and both HPF and LPF characteristics. The parameters illustrated in Figure $\mathbf{2 B}$ are listed in Table 1. The details of the amplifier transfer function and the corner frequencies are described in Supplementary Material.

\section{STIMULATION CIRCUITRY}

In this system, each electrode can be switched between recording and stimulation, and stimulation of neuronal cells can be realized by voltage pulses. The stimulus signal is approximately 500 times the recorded signal or larger, and changes very rapidly. It therefore causes extremely large artifacts, which can totally obscure spikes and saturate the high-gain recording amplifier. To avoid this, the recording inputs should be disconnected during stimulation. However, if the input level of the recording amplifier is floating during stimulation, its output is highly unstable and this will cause additional transients. Thus, the input level should be kept fixed at some value. Our design goal was to fix it at the input level that exists just before the stimulus is applied, which is nonzero because of the DC offset between electrode and electrolyte. This DC offset is also important to consider in generating reliable stimulation of known amplitude. The standard method of electrical stimulation is to apply constant voltage (or current) pulses to the electrode through an isolating circuit. If, however, there is a nonnegligible potential difference between stimulus and reference electrodes, then direct application of the stimulation pulse between electrode and the reference electrode produces an error in the stimulus applied at the interface of the electrode terminal. Therefore, a reasonable design goal is to add the stimulation pulse to the initial offset level (Jimbo et al., 2003).

To reduce stimulus transients, there is one further practical factor that should be considered. The electrode/electrolyte interface has a large capacitive component, which is charged by the electrical stimulation. Because of the slow time constant of the cellular/tissue interface and the series resistance attached to it, it is not discharged rapidly. As a result, the potential difference between stimulation electrode and reference electrode is not identical before and after the stimulus. This causes another transient when the electrode is reconnected to the recording input 
A
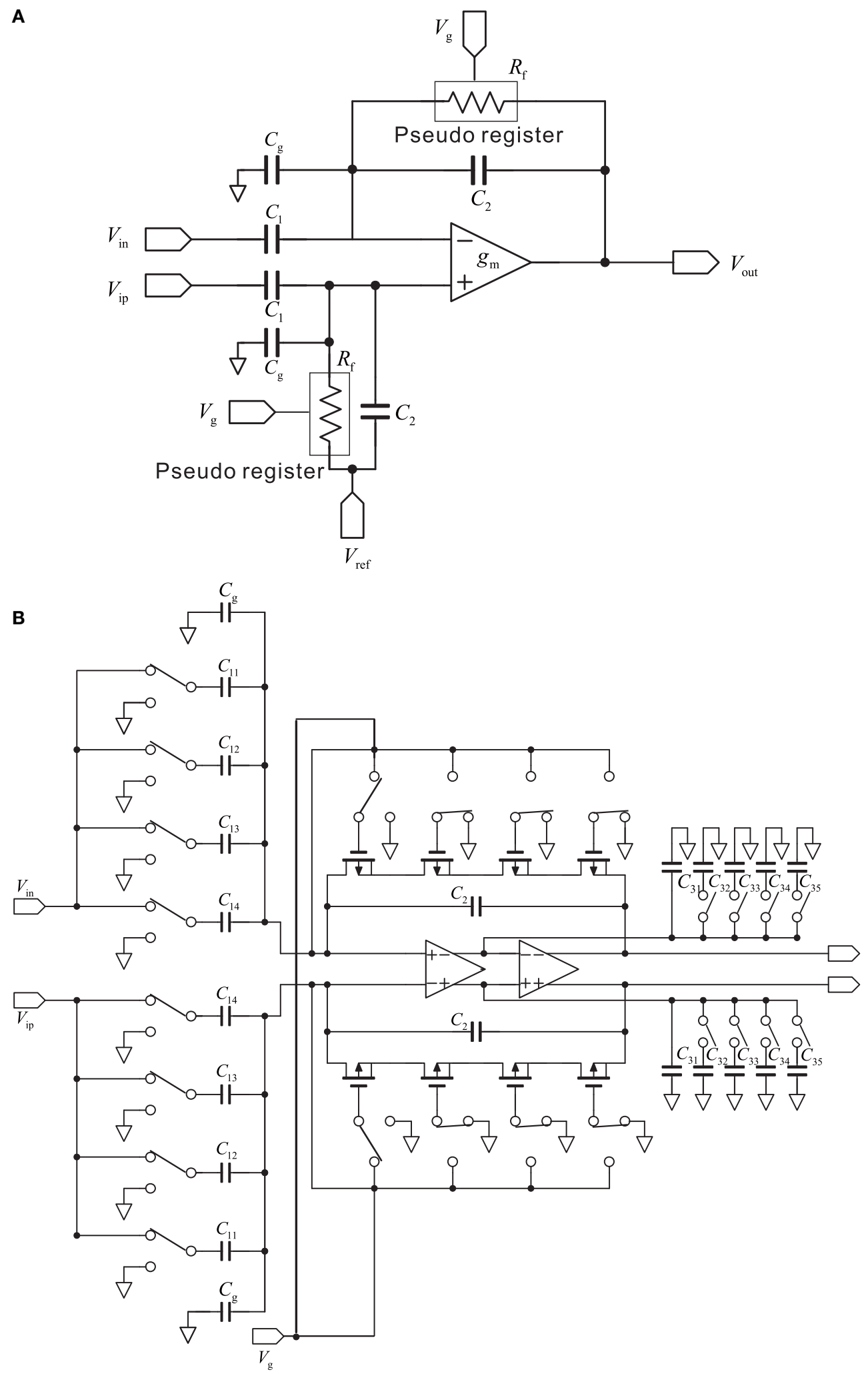

FIGURE 2 | (A) Schematic of a standard capacitive-feedback amplifier for neural signal amplification of each channel. The mid-band gain $A_{m}$ is set by $C_{1} / C_{2}$, and $g_{m}$ is the transconductance of the operational transconductance amplifier (OTA). MOS-bipolar transistors act as pseudoresistors and are represented by simple resistors $R_{\mathrm{f}}$. Gate voltage of the pseudoregister, stray capacitance, and reference voltage are respectively represented by $V_{g}, C_{g}$, and $V_{\text {ref. }}$ (B) Schematic of the low-noise amplifier with variable corner frequencies of low-and high-pass filters (see text for more detail). 
Table 1 | Summary of the parameters in the low noise amplifier.

\begin{tabular}{lcc}
\hline Elements & Parameters & Unit(s) \\
\hline$C_{11}$ & $1.00 \times 10^{-12}$ & $\mathrm{~F}$ \\
$C_{12}$ & $5.00 \times 10^{-13}$ & $\mathrm{~F}$ \\
$C_{13}$ & $5.00 \times 10^{-13}$ & $\mathrm{~F}$ \\
$C_{14}$ & $3.00 \times 10^{-12}$ & $\mathrm{~F}$ \\
$C_{2}$ & $5.00 \times 10^{-14}$ & $\mathrm{~F}$ \\
$C_{31}$ & $2.70 \times 10^{-12}$ & $\mathrm{~F}$ \\
$C_{32}$ & $1.00 \times 10^{-12}$ & $\mathrm{~F}$ \\
$C_{33}$ & $1.90 \times 10^{-12}$ & $\mathrm{~F}$ \\
$C_{34}$ & $5.80 \times 10^{-12}$ & $\mathrm{~F}$ \\
$C_{35}$ & $1.67 \times 10^{-11}$ & $\mathrm{~F}$ \\
$R_{f, 1}$ & $3.20 \times 10^{10}$ & $\Omega$ \\
$R_{f, 2}$ & $3.20 \times 10^{11}$ & $\Omega$ \\
$R_{f, 3}$ & $3.20 \times 10^{12}$ & $\Omega$ \\
$R_{f, 4}$ & $3.20 \times 10^{13}$ & $\Omega$ \\
$g_{m}$ & $1.80 \times 10^{-5}$ & $\mathrm{~S}$ \\
\hline
\end{tabular}

after stimulation, which can easily be large enough to produce unwanted excitation. Therefore, a further design goal was to include a low-impedance discharge path for the injected electrical charge. To realize this, we previously constructed a system for MEA-based multisite stimulation (Jimbo et al., 2003). However, the design was based on discrete off-chip components and the size was relatively larger than that of an animal brain. In contrast, Heer et al. reported a CMOS metal-electrode-based microchannel system for stimulation and recording with neural cells in vitro (Heer et al., 2007). In this study, although our design concept was similar to that of Heer et al. (2007), our goal was to apply it not only to stimulation and recording of neurons in vitro but also to those in vivo for our future work.

For one unit of the 64-channel stimulation system, Figure 3A shows the block diagram of the interface circuit. The circuit in the stimulation unit incorporates a voltage amplifier, low charge injection switches, and a sample-and-hold circuit. The timing of the applied stimulus and of the three switches $\left(\mathrm{SW}_{\mathrm{Stim}}, \mathrm{SW}_{\mathrm{S} / \mathrm{H}}\right.$, and $\left.\mathrm{SW}_{\text {Add }}\right)$ is controlled, so that the voltage of each electrode before stimulation is fixed (Jimbo et al., 2003). In addition, the stimulus waveforms are created by the waveform generator (DAC) on the CMOS IC chip, according to the information from the DI1 with specific parameters. During recording, the DC offset level at each electrode is continuously monitored and stored in the sample-and-hold circuit (Figure 3Aa).

Before stimulation, $\mathrm{SW}_{\mathrm{Add}}$ and $\mathrm{SW}_{\text {Stim }}$ are "Off," and the DAC value is zero (Figure $\mathbf{3 A b}$ ). In this state, therefore, the stimulation circuit is not active and the amplifier processes neuronal signals. Prior to stimulus application, in the first step of a stimulation process, the amplifier gets disconnected from the electrode by setting the record switch $\left(\mathrm{SW}_{\mathrm{Rec}}\right)$ to "Off," and the amplifier input potential is then held at a constant value by a capacitor. When SW $_{\text {Add }}$ is turned on, the DC offset $\left(V_{\text {add }}\right)$ stored by the capacitor is added to the stimulus voltage in the stimulation mode. Thus, once the amplifier is disconnected, the stimulation signal can be applied to the electrode. In this phase, $\mathrm{SW}_{\text {Stim }}$ is set to "On" after a short delay $(\Delta t)$, and the DAC value can be updated, depending on the required stimulation pattern and time slots (i.e., time divisions during a stimulation period for stimulus pattern update). After the stimulus pulse, $\mathrm{SW}_{\text {Stim }}$ and $\mathrm{SW}_{\text {Add }}$ are set to "Off," and the electrode residual voltage is discharged during one time slot. Once the discharging is finished, $\mathrm{SW}_{\mathrm{Rec}}$ is set to "On," and the system is back in the sensing (recording) mode. These operations are necessary to minimize the damage to the neural cells and suppress the stimulation artifacts.

To realize multisite stimulation using a small number of input/output (I/O) pads, we designed a serial-to-parallel digital converter and a 5-bit DAC for each of 64 channels (Figure 3B). This circuit system can supply a set of voltage time series to a 64-channel pad connected to each electrode. The digital data converted from the serial digital data are stored in a flip-flop circuit of each channel, and a 64-channel stimulus signal pattern is applied simultaneously at a clock-timing from 1.6 to $16 \mathrm{kHz}$. As a result, this clock-timing determines the pulse width and stimulation patterns, including interpulse intervals. Thus, this system realizes a programmable stimulus pattern both spatially and temporally. The maximum and minimum stimulation signal voltage amplitude employed with a biphasic/monophasic voltage waveform is $\pm 2.5 \mathrm{~V}$. The reference voltage is created simply with a resistance ladder. Therefore, the peak voltage is tunable. In the stimulation protocol, the mode is first switched from the sensing mode to the stimulation mode, then a reset signal is applied to the serial-toparallel controller, and finally, continuous serial digital data are converted to an analog voltage waveform for stimulation at any channel with any timing (cf. timing chart in Figure 3Ab). The circuit speed is sufficiently high and the time interval of switching is less than $62.5 \mu \mathrm{s}$; therefore, a waveform generator system controlled with serial digital data can be easily scaled to a 64-channel system.

\section{TOTAL SYSTEM CIRCUITRY AND OPERATION}

As mentioned above (Figure 3B), the CMOS IC chip consists of 64-channel LNAs designed to sense small neural signals and stimulation signal formation circuits. This chip makes it possible to realize simultaneous multisite stimulation and neuron sensing through multi-electrodes in vitro. Before the operation of the system, parameters of pulse patterns and timings of switching between the sensing mode and the stimulation mode are transferred into the chip from custom-made PC software via the FPAG board. The timing is controlled with an on-chip signal synchronized by the main clock $(48 \mathrm{MHz})$ that is supplied from the FPGA card (SX-Card 6, Prime Systems, Inc., Japan). The transition time from the recording mode to the stimulation mode is about $0.063-1.0 \mathrm{~ms}$, which corresponds to the stimulation time-slot clock frequency at $1.0-16 \mathrm{kHz}$. When the CMOS IC chip is applied to the MEA measurement system, it takes about $1.2 \mathrm{~ms}$ in addition to the switching time to sense the evoked neural spikes because the capacitance at the electrode/electrolyte interface discharges toward the initial state (Jimbo et al., 2003). Thus, evoked spikes after the stimulus offset can be recorded after $1.3-2.2 \mathrm{~ms}$, and $1.3 \mathrm{~ms}$ after stimulus offset is the minimum latency for recording neural spikes. The recording unit block for neural signal sensing and the stimulation unit block for handling stimulation signals are integrated on the CMOS IC chip 


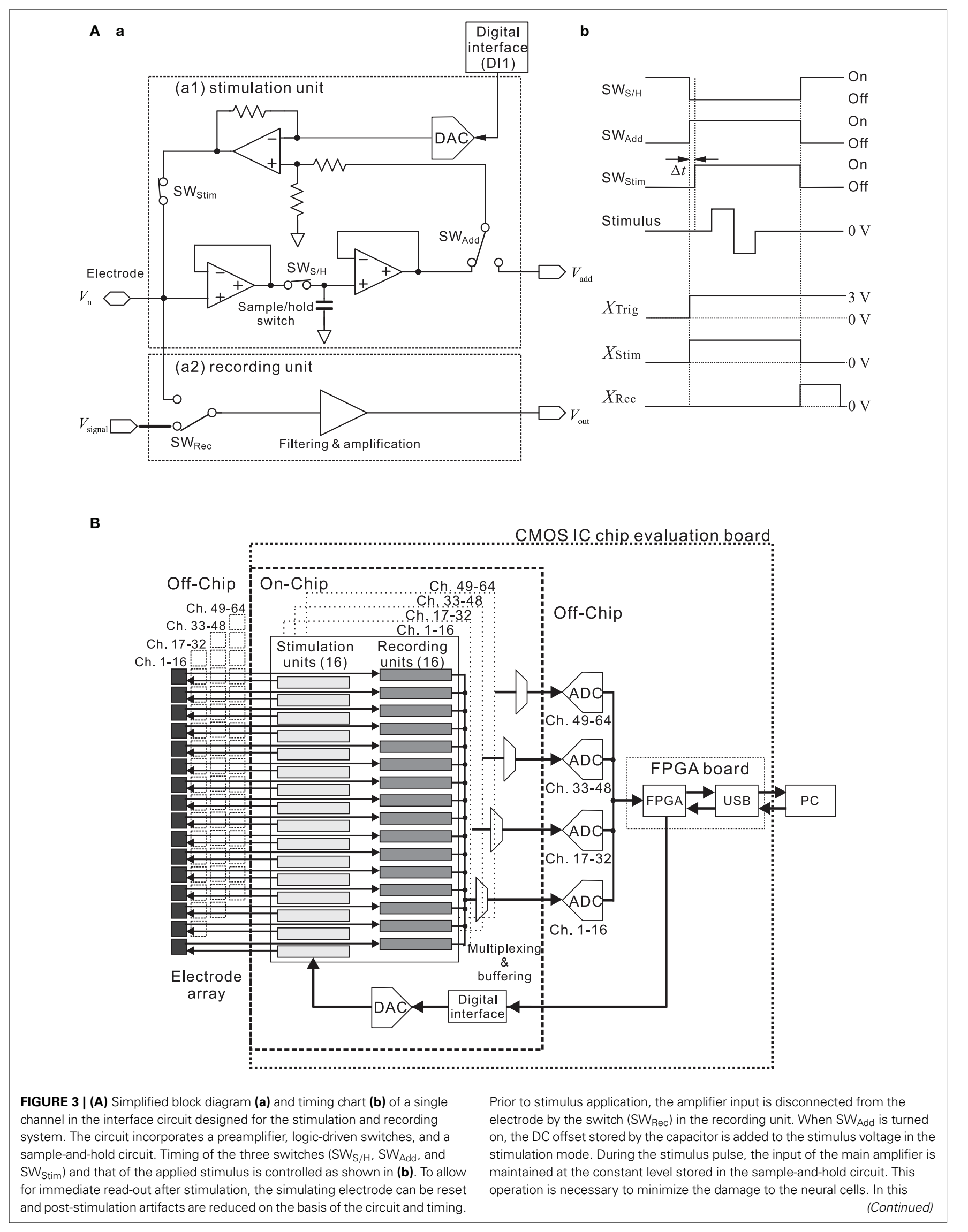




\section{FIGURE 3 | Continued}

system, each switch control signal is triggered by a different signal ( $X_{\text {Trig, }}$ $X_{\text {Stim }}$, and $\left.X_{\text {Rec }}\right)$, and all are transmitted through the FPGA card from a PC. Before $\mathrm{SW}_{\mathrm{Stim}}$ turns on, there exists a short time delay $\Delta t$ after $\mathrm{SW}_{\text {Add }}$ is turned on. The time delay depends on the stimulation time slot (time divisions of the stimulus pattern) and ranges from 6.25 to $62.5 \mu \mathrm{s}$. In addition, $X_{\text {Trig }}$ can be replaced with a signal provided by an external trigger source (cf.
Figure 1). (B) Simplified circuit diagram of the designed system. The CMOS IC chip consists of 64-channel low noise amplifiers (recording units), designed to sense small neural signals, and stimulation signal formation circuits. At the system level, our approach to the multiple sensory channel system consists in sharing a fast digitizer between the multiple channels. That is, 16 recording units are directed toward the analog-to-digital converter (ADC), employing time-division multiplexing in the analog domain.
(Figure 3B). After they are sensed by the amplifiers, neural signals from 16 electrodes are multiplexed and transferred into each 16-bit ADC on the CMOS IC chip evaluation board (Figure 1). After that, the four ADCs transfer the digital data to the FPGA card, and the data are subsequently transferred to a PC.

\section{CHIP DESIGN AND FABRICATION}

The chip was fabricated using industrial $0.25-\mu \mathrm{m}$ CMOS mixedsignal technology (TMSC, Taiwan). The micrograph of the $5.0 \times$ $5.0 \mathrm{~mm}$ chip (Figure 4A) shows four identical main areas, each of which is assigned 16 channels. Each block in the main areas comprises three sub-blocks; the 16-channel circuit, the buffer circuit, and the bias circuit sub-blocks. The central control circuit is shared by all 64 channels and is located in the bottom central area of the chip (Figure 4Ab). The design of the 16-channel circuit block is modular in that the same circuit unit is repeated 16 times (Figure 4A) and each row of 16 units includes the recording unit of the LNA and the stimulation unit (Figure 3A).

\section{FUNCTIONS OF FPGA CARD AND CMOS IC CHIP EVALUATION BOARD}

The large data volumes to be processed and the desired lowlatency time require high-performance communication and signal processing capabilities between the CMOS IC chip and a PC. In our system, digital logic components are located on the FPGA on the CMOS IC chip evaluation board and connecting to the CMOS IC chip (Figures 1, 4B). In the two DIs (DI1 and DI2) of the CMOS IC chip, the on-chip digital logic runs at $2.0 \mathrm{MHz}$ and $2.5 \mathrm{~V}$ and serves two purposes: (i) operation control in DI1 and (ii) parameter setting in DI2. Firstly, via the operation control DI (DI1), it performs control tasks that include multiplexing, electrode selection for stimulation, and reset of single electrodes, and it also contains the successive-approximation registers of the ADC. Secondly, via the parameter setting DI (DI2), it provides the chip interface to the FPGA. DI2 also stores amplifier settings, including total gain, corner frequencies of LPF and HPF, and pulse pattern stimulation settings. In the CMOS IC chip, these DIs use four lines for the data readout, four lines for parameter settings, two lines for operational control, and four lines for clocks (14 lines in total). All signals are synchronous to the $48-\mathrm{MHz}$ master clock supplied from the FPGA card. In this study, we used a commercially available FPGA card (SX-Card6, Prime Systems Inc., Japan). The card provides the FPGA hardware platform for system designs that demand high performance, serial connectivity, and advanced memory interfacing. It is powered by the Xilinx Spartan-6 FPGA device (XC6SLX75-3FGG676C, Xilinx, USA) and supported by industry-standard peripherals, connectors, and interfaces. Briefly, to manage the data input and output rate of $40 \mathrm{MB} / \mathrm{s}$, the FPGA runs at $48 \mathrm{MHz}$ in conjunction with a USB 2.0 interface chip (CY7C68013A-56LFXC, Cypress, USA) and utilizes 1 GB DDR2 SDRAM (MT47H64M16HR-3, Micron, USA) (see the supplier's web site for more details). The output of the CMOS IC was sampled at $15.6 \mathrm{kHz}$ and 16-bit resolution by the ADCs of the CMOS IC chip evaluation board, and the sampled data were transferred into a PC via the FPGA card. The sampling frequency of $15.6 \mathrm{kHz}$ was the maximum we tested in the experiments below.

\section{MEASURING FREQUENCY CHARACTERISTICS OF THE AMPLIFICATION CIRCUIT}

To measure frequency characteristics of the chip circuitry, the frequency of sinusoidal signals was controlled between 0.10 and $100,000 \mathrm{~Hz}$ using a function generator $(33120 \mathrm{~A}$, Agilent Technologies Inc., USA) or a frequency response analyzer (FRA 5022, NF Co., Japan). The output signals were analyzed using the frequency response analyzer and a spectrum analyzer (R9211C, Advantest Co., Japan). In addition, to illustrate several analog signal waveforms, the output signals were sampled at $200 \mathrm{kHz}$ using an NI PCI-6259 DAQ card (National Instruments, USA) and custom data acquisition software written in MATLAB R2012 (Mathworks, USA).

\section{SIMULATION OF CIRCUIT MODELS AND DATA ANALYSIS FOR SPIKE DETECTION}

An analysis of the designed circuit, including MOS transistors, was performed quantitatively using the Berkeley short-channel IGFET model (BSIM, ver. 4.6.0) simulator (Sheu et al., 1987). In the simulation, MOSFET noise sources included flicker noise, channel thermal noise, induced gate noise, and their correlations. A complete list of the noise model parameters and explanations are described in the user's manual of the BSIM4.6.0 MOSFET model. In this study, a lower or higher corner frequency of a filter transfer function is defined as the frequency at which the filter gain falls by $-3.0 \mathrm{~dB}$ from the mid-band gain.

To detect neural spikes immediately after stimulation, each recorded voltage transient was first approximated as a $p$-th order polynomial function to minimize the error between the function and the voltage trace. Then, the approximated curve was subtracted from the voltage trace, so that a targeted signal including spikes was obtained. Usually, 5-th to 6-th order polynomial functions were enough to approximate the voltage transients (i.e., $p=5$ or 6$)$ to avoid overfitting. After the subtraction, spikes were detected when the signal exceeded a threshold. The threshold level for detecting spikes was set at 3.0 times the standard deviation (SD) of the baseline noise (Tateno and Robinson, 2006). The artifact subtraction and spike detection were carried out on off-line in a PC. In all figures, error bars are represented as SDs. By an unpaired $t$-test, a $p$-value less than 0.01 was considered statistically significant. Data are presented as the mean $\pm S D$. 
A

a

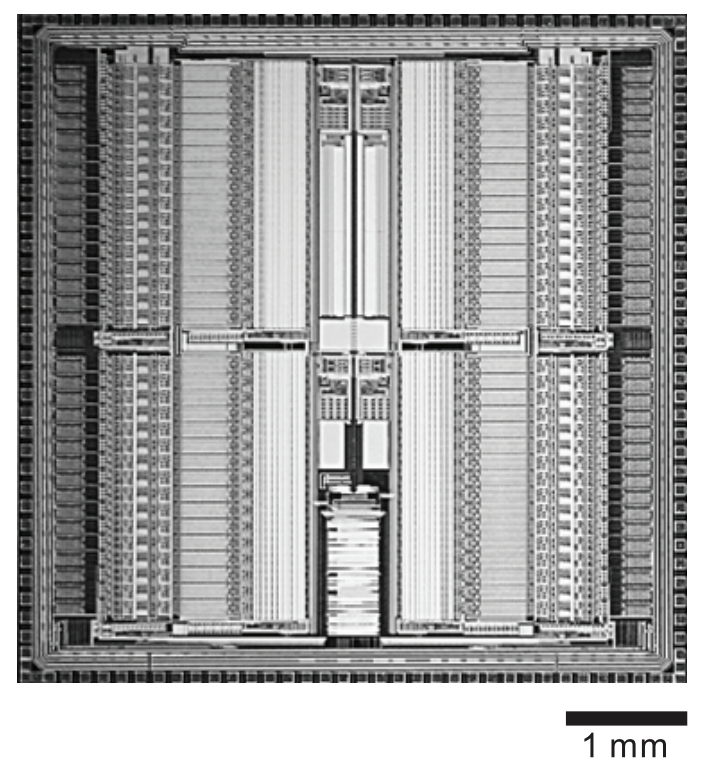

b

Ch-1 circuit block Ch-17 circuit block

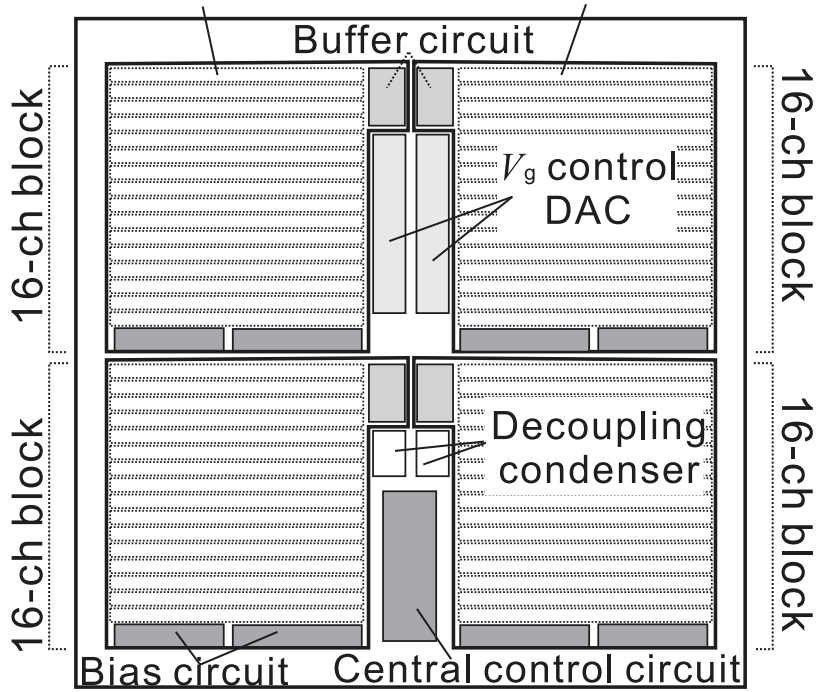

$1 \mathrm{~mm}$

B LSI chip evaluation board

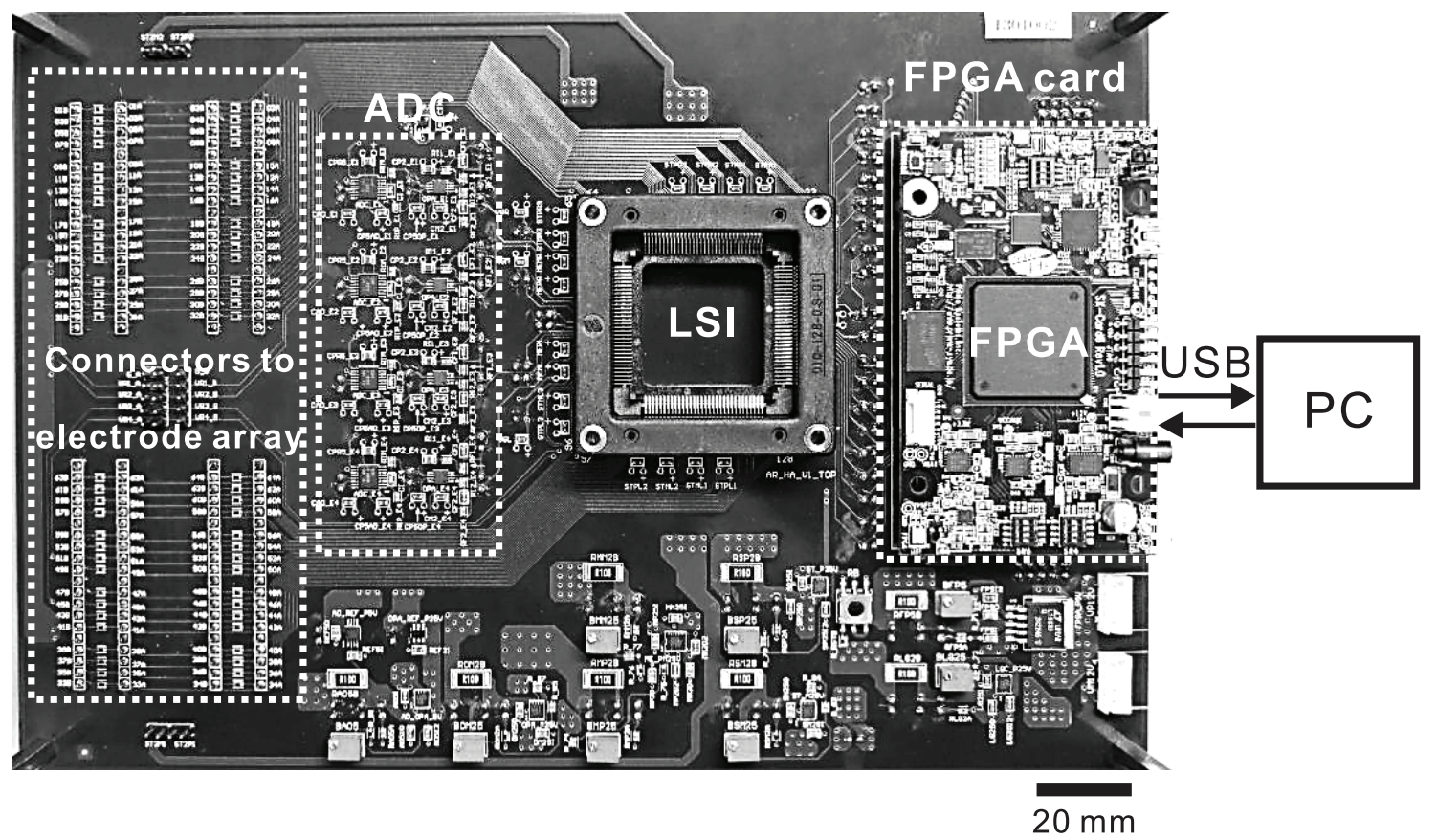

FIGURE 4 | (A) Micrograph of the CMOS IC chip (a) and schematic (b). The chip comprises 4 buffer circuits, 8 bias circuits, $2 V_{\mathrm{g}}$ control ADCs, and 4 recording and stimulation blocks including 16 identical units per block. (B)
Photograph of the CMOS IC chip evaluation system board. The board includes connectors to an MEA substrate, ADCs, a FPGA card, and an I/O interface to a PC.

\section{RAT CORTICAL CELL CULTURE}

To test our CMOS IC system, we used two in vitro preparations: rat cortical cultures and mouse acute brain slices. All animal experiments described below were carried out in accordance with the National Institutes of Health Guidelines for the Care and Use of Laboratory Animals, and with approval of the Institutional Animal Care and Use Committee of Hokkaido University. We used a culture method similar to that described in previous 
studies (Tateno and Jimbo, 1999; Tateno et al., 2005), with slight modifications. Briefly, cortical tissue of newborn Wistar rats (Japan SLC Inc., Japan) was finely chopped, digested using a papain dissociation system (Worthington Biochemical Co., USA), and mechanically dissociated with trituration. Then, cortical cells were plated on multielectrode substrates (MED-P210A and MED-P515A, Alpha MED Scientific, Japan), coated with laminin (Cat. 354232, BD Biosciences, USA) and poly-D-lysine (P2636, Sigma-Aldrich, USA), and cultured in neurobasal medium (Cat. 21103-049, Life Technologies Co., USA) containing 2\% B27 supplement (Cat. 17504044, Life Technologies Co.), 1\% GluataMax (Cat. 35050-061, Life Technologies Co.), $2.5 \mathrm{mg} / \mathrm{ml}$ insulin (Cat. 10516, Sigma-Aldrich), and 5-40 U/ml penicillin-streptomycin (Cat. P0781, Sigma-Aldrich). The density of cells in the culture was around $15,000 \mathrm{cell} / \mathrm{mm}^{2}$. The medium was changed twice a week. All the activity in the cultured networks was recorded in vitro from the 30th to the 45th day. Before the measurements with our CMOS-IC based system, the MEAs were tested using commercially available amplifiers (MED-A64HE1 and MED-A64MD1, Alpha MED Scientific Inc., Japan). The material of working and reference electrodes on the MEAs was platinum, and each electrode was covered with platinum black. Contact electrodes for the connectors were also located on the MEA substrate, and the material was indium tin oxide (ITO) with no platinum black. Although during development the electrophysiological properties of cortical neurons change according to the expression of receptors and voltage-dependent ion channels (Luhmann and Prince, 1991), rat cortical neurons previously showed stable patterns of continuous synchronized firing when cultured for the period used in the present study (Kamioka et al., 1996).

\section{ACUTE MOUSE SLICES INCLUDING THE HIPPOCAMPUS OR THE AUDITORY CORTEX}

To record FPs from brain slices in vitro, transverse or coronal slices (400- $\mu \mathrm{m}$ thick) including the hippocampus or auditory cortex were prepared from 10- to 15-week-old C57BL/6J and SAMR1 mice (Japan SLC Inc., Japan). In the experimental results presented here, no critical differences between the two mouse strains were found. Details from the electrophysiological experiments will be described elsewhere in the near future. Briefly, chilled artificial cerebrospinal fluid (ACSF) saturated with $95 \% \mathrm{O}_{2}$ and $5 \% \mathrm{CO}_{2}$ mixed gas was prepared for use in slicing a brain block including the hippocampus and the cortex. The ACSF contained (in mM) $119 \mathrm{NaCl}, 2.5 \mathrm{KCl}$, $2.5 \mathrm{CaCl}_{2}, 1.3 \mathrm{MgSO}_{4}, 1.0 \mathrm{NaH}_{2} \mathrm{PO}_{4}$, and 11.0 D-glucose $(\mathrm{pH}$ $=7.4$ ). A mouse was deeply anesthetized with halothane and decapitated. Then, slices were cut with a tissue slicer (Linear Slicer Pro7, D.S.K., Japan) in the chilled ACSF. The slices were recovered in a submerged-type holding chamber at $28^{\circ} \mathrm{C}$ in a water bath for at least $2 \mathrm{~h}$ before recording. All electrophysiological recording in brain slices was performed with ACSF perfusion and the mixed gas supply from the top of the recording chamber in an incubator (APC-30, Asteck Co., Japan) maintained at $28.0^{\circ} \mathrm{C}$. The perfused ACSF solution was also warmed and maintained at $28.0^{\circ} \mathrm{C}$, so that the bath was always kept at the same temperature. While recording, slices were plated on multielectrode substrates (MED-P210A and MED-P515A, Alpha MED Scientific, Japan) and covered with a nylon mesh and a stainless slice anchor.

Induction of short- and/or long-term potentiation generally involves short, high-frequency presynaptic stimulation consisting of a series of bipolar pulse trains, termed "tetanic stimulation." Typical frequencies used to induce such potentiations range $10-250 \mathrm{~Hz}$, and the stimulation contains 20-100 bipolar short pulses. In this particular study, we used a frequency of $125 \mathrm{~Hz}$ (i.e., the interpulse interval was $8 \mathrm{~ms}$ ) and trains of 100 pulses as the tetanic stimulation (TS).

\section{ACOUSTIC SOUND AND SIGNAL PROCESSING BOARD}

A polyvinylidene difluoride (PVDF) film and a custom-made electric circuit board (Tateno et al., 2013) were used to process acoustic sound and send a trigger signal to the CMOS IC system. The film was used as a piezoelectric sensor to convert acoustic sound pressures into corresponding electric signals (Shintaku et al., 2010). A sinusoidal acoustic wave sound (tone burst) was applied to the sensor device from a speaker (FT96H, Fostex, Japan) located $50 \mathrm{~mm}$ away at a $45^{\circ}$ angle. To produce a constant sound pressure level (SPL) with a precision of \pm 0.1 (SD) $\mathrm{dB}$ at various frequencies, the speaker was previously calibrated using a measuring amplifier (Type 2636, Brüel \& Kjær, UK). The frequency was fixed at $4.9 \mathrm{kHz}$, which was a resonance frequency of a readout electrode on the film, using a function generator (WF 1973, NF Co., Japan) or a frequency response analyzer (FRA 5022, NF Co., Japan). The duration of the sound waveform was 15 or $20 \mathrm{~ms}$ with a $75 \mathrm{~dB}$ SPL, and a 4 -ms rise/fall time was used. A Hanning window was usually used to obtain a slow onset. For more details about the sensor device and the experimental setup, see Tateno et al. (2013).

\section{RESULTS}

\section{CIRCUIT FREQUENCY RESPONSE CHARACTERISTICS}

Measured transfer functions that characterize frequency response properties of the LNAs and filters we designed are shown in Figure 5. The LNA was designed to give mid-band voltage gains $\left(G_{\mathrm{d}}\right)$ of $40.0,32.0,29.5$, and $26.0 \mathrm{~dB}$ via the gain selector switch, and the specific gain could be selected via the DI2 from a PC. For five evaluated chips, the measured mid-band gains $\left(G_{\mathrm{e}}\right)$ were $39.4 \pm 0.6,31.8 \pm 0.7,29.6 \pm 0.8$, and $26.2 \pm 0.8($ mean $\pm \mathrm{SD})$ from largest to smallest (Figure 5A and Table 2), with the average very similar to that of our design specification.

As with the amplifier mid-band gain, it is possible to select the higher corner frequencies (HCFs, $f_{\mathrm{H}}$ ) of the LPF and the lower corner frequencies (LCFs, $f_{\mathrm{L}}$ ) of the HPF (Figures 5B,C). The measured result shows that the tunable HCFs of the LPF were $8.25 \pm 0.78,5.50 \pm 0.97,3.88 \pm 0.62,1.89 \pm 0.37$, and $0.80 \pm$ $0.11 \mathrm{kHz}$. Although the proposed amplifier achieved five different HCFs, each measured $f_{\mathrm{H}}$ was smaller than the corresponding $f_{\mathrm{H}}$ of the designed LPF (cf. Table 2). For this reason, we consider that parasitic gate capacitances at the OTA output terminals are likely to be responsible for the discrepancy between the designed and measured $f_{\mathrm{H}}$. Although we could not realize the exact design values of $f_{\mathrm{H}}$, the ability to adjust the HCFs to target signals can be useful for neural signal recording. 

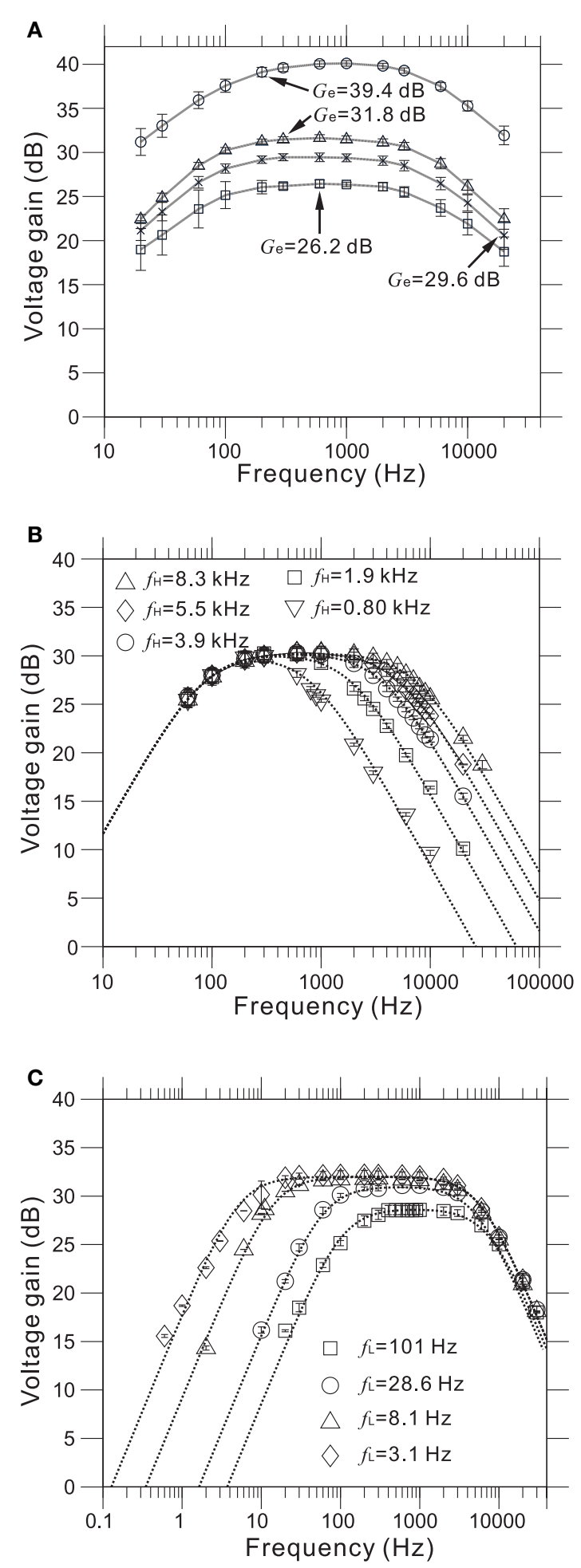

FIGURE 5 | (A) Measured frequency response functions (FRFs) of four mid-band voltage gains. The average measured gains $\left(G_{e}\right)$ are 26.2, 29.6, 31.8, and 39.4 dB from smallest to largest (see also Table 2). (B) Measured FRFs of five low-pass filters (LPFs) with different higher corner frequencies $\left(\mathrm{HCFs}, f_{\mathrm{H}}\right)$. The average measured HCFs are $0.80,1.9,3.9,5.5$, and $8.3 \mathrm{kHz}$. The dotted curves represent the corresponding FRFs obtained from

(Continued)

\section{FIGURE 5 | Continued}

approximated transfer functions described in Supplementary Material. (C) Measured FRFs of four LPFs with different lower corner frequencies (LCFs, $\left.f_{\mathrm{L}}\right)$ and the same gate voltage $\left(V_{\mathrm{g}}=-200 \mathrm{mV}\right)$. The average measured LCFs are 3.1, 8.1, 28.6, and $101 \mathrm{~Hz}$. The dotted curves represent the FRFs obtained from approximated transfer functions.

Table 2 | Summary of measured gain and low and high cutoff frequencies in the CMOS IC chip.

\begin{tabular}{|c|c|c|}
\hline Gain & $\begin{array}{l}\mathrm{G}_{\mathrm{d}} \text {, Designed } \\
\text { at } 1 \mathrm{kHz}(\mathrm{dB})\end{array}$ & $\begin{array}{c}\mathrm{G}_{\mathrm{e}}, \text { Measured at } 1 \mathrm{kHz} \\
\left({ }^{*} n=5\right) \text { Mean } \pm S D(\mathrm{~dB})\end{array}$ \\
\hline & 40.0 & $39.4 \pm 0.6$ \\
\hline & 32.0 & $31.8 \pm 0.7$ \\
\hline & 29.5 & $29.6 \pm 0.8$ \\
\hline & 26.0 & $26.2 \pm 0.8$ \\
\hline
\end{tabular}

Higher corner frequency Designed $(\mathrm{kHz}) \quad$ Measured $\left({ }^{*} n=5\right)(\mathrm{kHz})$ $\left(f_{H}\right)$ of low pass filters

$\begin{array}{ll}10 & 8.25 \pm 0.78 \\ 7.5 & 5.50 \pm 0.97 \\ 5.0 & 3.88 \pm 0.62 \\ 2.5 & 1.89 \pm 0.37 \\ 1.0 & 0.80 \pm 0.11\end{array}$

Lower corner frequency $\left(f_{L}\right)$ of high pass filters

\begin{tabular}{cr} 
Designed $(\mathrm{Hz})$ & Measured $\left({ }^{*} n=5\right)$ \\
\hline 100 & $101.3 \pm 3.5$ \\
10 & $28.6 \pm 4.3$ \\
1.0 & $8.1 \pm 3.1$ \\
0.1 & $3.1 \pm 3.0$
\end{tabular}

${ }^{*} n=5$ represents that five amplifiers in different chips were tested.

Furthermore, when the gate voltage $\left(V_{\mathrm{g}}\right)$ of MOSFETs was set to be $-200 \mathrm{mV}$, Figure 5C shows that the LCFs of the HPF were $101.3 \pm 3.5,28.6 \pm 4.3,8.1 \pm 3.1$, and $3.1 \pm 3.0$. However, the designed LCFs were smaller than the measured ones, excluding the LCF of $100 \mathrm{~Hz}$ (Table 2). In addition, as dotted lines in Figure 5C indicate the approximated transfer functions of the HPFs, the measured mid-band gains were slightly reduced in a range from 0.5 to $5.0 \mathrm{~dB}$ from the designed gain of $32 \mathrm{~dB}$. Because the discrepancy between the measured and designed LCFs of the HPFs is likely to be a variance of $R_{\mathrm{f}}$, we could not achieve LCFs of under $1 \mathrm{~Hz}$ in this chip design and fabrication. Also, we can see that the variance of LCFs in individual chips was relatively larger (cf. Table 2).

Furthermore, values of the gate voltage $V_{\mathrm{g}}$ profoundly affected the transfer functions, especially those of the HPF. Therefore, an appropriate choice of $V_{\mathrm{g}}$ value is necessary for the desired transfer function. For five selected values of the adjustable $V_{\mathrm{g}}$ (e.g., $V_{\mathrm{g}}=-500,-400,-300,-200$, and $-100 \mathrm{mV}$ ) at a mid-band gain of $32 \mathrm{~dB}$, Figure 6Aa shows the frequency responses corresponding to the HPF with a measured LCF of $f_{\mathrm{L}}=3.1 \mathrm{~Hz}$ (Figure 5C) when $V_{g}=-200 \mathrm{mV}$. Also, Figure 6Ab shows the dependency of measured $f_{\mathrm{L}}$ against $V_{\mathrm{g}}$ for two HPFs, in the two cases of the average measured $f_{\mathrm{L}}=3.1$ and $28.6 \mathrm{~Hz}$ for an identical gate voltage $\left(V_{\mathrm{g}}=-200 \mathrm{mV}\right)$ in Figure 5C. For the range of 
$V_{\mathrm{g}}$ from -500 to $0 \mathrm{mV}$, we can see that measured $f_{\mathrm{L}}$ is saturated to around $3 \mathrm{~Hz}$ (Figure 6Ab). This result indicates that the value of $f_{\mathrm{L}} \sim 3 \mathrm{~Hz}$ is the smallest LCF of the amplifier, thus limiting the chip design in terms of the LCFs of the HPF. However, in this study, the smallest LCF of around $3 \mathrm{~Hz}$ is sufficient for recording local FPs of in vitro preparations (see Results below).

In short summary, for each of four 16-channel groups, midband gains, HCFs of the LPF, and LCFs of the HPF are adjustable in any combinations for experimental purposes, allowing for selective measurement of specific target signals, including neural spikes, LFPs, and their combination.

For our five LNAs at the measured $31.8 \mathrm{~dB}$ gain, Figure 6B shows the average measured output and input-referred noise spectra superimposed with the corresponding spectra of a circuit simulation for a frequency range of $1 \mathrm{~Hz}$ to $100 \mathrm{kHz}$. The circuit simulator used was the Berkeley Short-channel IGFET Model (BSIM4.6.0) (Sheu et al., 1987; see also Materials and Methods). Additionally, the input-referred noise spectra (Figure 6Bb) were obtained by dividing the output noise spectra (Figure 6Ba) by the response function of the amplifier gain. The results demonstrate good agreement between the measured and simulated curves in frequencies below $200 \mathrm{~Hz}$, while a small discrepancy can be seen in the higher frequencies. This indicates that to reduce the higher frequency components, an appropriate selection of LCFs in the HPF is effective for obtaining target signals with a good signal-to-noise $(\mathrm{S} / \mathrm{N})$ ratio during the recording of extracellular potentials. For five LNAs, the measured thermal noise level was $93.8 \mathrm{nV} / \sqrt{\mathrm{Hz}}$. Integrating under the area of the measured curve from $10 \mathrm{~Hz}$ to $100 \mathrm{kHz}$ (Figure 6Bb) yielded a total input-referred noise of $10.1 \pm 0.2 \mu \mathrm{V}_{\mathrm{rms}}$ (Table 3), while the simulated result was $9.7 \mu \mathrm{V}_{\mathrm{rms}}$. This is comparable to the thermal noise of the platinum electrodes in physiological saline solution, which was measured to be $6.19 \mu \mathrm{V}_{\mathrm{pp}}$ for $50 \times 50 \mu \mathrm{m}$ MEA electrodes in a band between $3.1 \mathrm{~Hz}$ and $8.2 \mathrm{kHz}$ in the experiments presented here, because lower and higher frequency components were removed by the bandpass filter. However, the total noise in
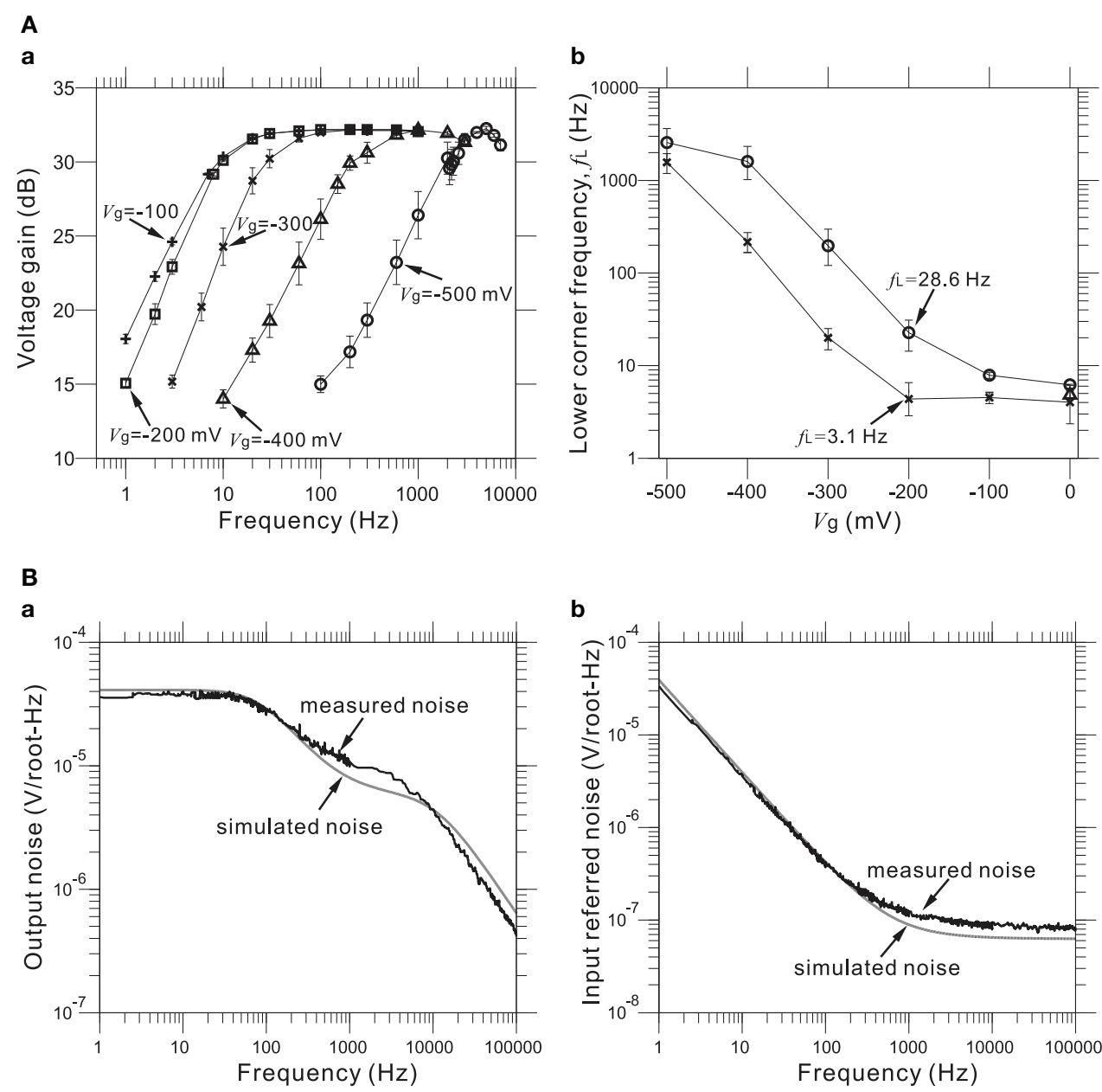

FIGURE 6 | (A) In (a), Measured FRFs at $31.8 \mathrm{~dB}$ gain with five different values (in $\mathrm{mV}$ ) of gate voltage $V_{\mathrm{g}}$ (i.e., from left to right, $V_{\mathrm{g}}=-100,-200$, $-300,-400$, and $-500 \mathrm{mV}$ ). Excluding the $V_{g}$ values, the other parameters of the FRFs are the same as those of the FRF of the HPF, with $f_{L}=3.1 \mathrm{~Hz}$ and $V_{\mathrm{g}}=-200 \mathrm{mV}$ (Figure $\mathbf{5 C}$ ). In (b), the relationships between gate voltages $V_{g}$ and LCFs of HPFs are shown for two parameter sets ( $x$ and $\circ$ ),

b

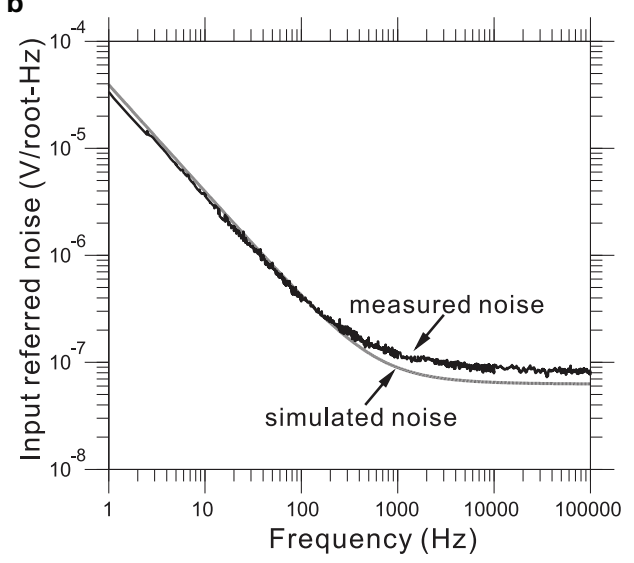

which respectively correspond to two HPFs with $f_{\mathrm{L}}=3.1 \mathrm{~Hz}$ and $f_{\mathrm{L}}=28.6 \mathrm{~Hz}$ at the identical $V_{\mathrm{g}}=-200 \mathrm{mV}$ (Figure 5C). (B) $\ln$ (a), measured and simulated output-noise spectra for a bandpass filter with a 31.8-dB gain, the smallest $L C F\left(f_{L}=3.1 \mathrm{~Hz}\right)$, the largest HCF $\left(f_{H}=8.3 \mathrm{kHz}\right)$, and the gate voltage $\left(V_{\mathrm{g}}=-200 \mathrm{mV}\right)$ are shown. Similarly, in (b), measured and simulated input-referred noise spectra are shown with the same parameter sets of (a). 
the actual measurements has been found to be dominated by the actual background activity of the neuronal culture, which in previous studies was often on the order of $10-30 \mu \mathrm{V}_{\mathrm{pp}}$ in some dense cultures over 30 days in vitro (Tateno and Jimbo, 1999; Tateno et al., 2005). Moreover, the measured power consumption of the chip was $1.4 \mathrm{~mW}$ at $2.5 \mathrm{~V}$ for 64 channels (i.e., $22 \mu \mathrm{W} /$ channel), a value relatively higher than those reported in other previous studies (Harrison and Charles, 2003; Hafizovic et al., 2007; Heer et al., 2007). The reason for this could be that the circuit functions not only during recording but also stimulation, for a maximum voltage pulse of $\pm 2.5 \mathrm{~V}$. The obtained results are listed in Table 3 .

\section{RECORDING AND STIMULATION IN RAT CULTURED CORTICAL NEURONS}

Neural networks originating from dissociated cortical tissue obtained from newborn rats at postnatal day 1 (P1) were cultured on MEA substrates. During development the electrophysiological properties of cortical neurons change according to the expression of receptors and voltage-dependent ion channels (Luhmann and Prince, 1991; Burgard and Hablitz, 1993). Over a period of around 30 days in vitro (DIV), rat cortical neurons cultured on such MEA substrates showed stable patterns of continuous synchronized burst firing (Kamioka et al., 1996; Watanabe et al., 1996). The arrangement of the multielectrodes used in this experiment is shown schematically in Figure 7A. Signals from spontaneously firing neurons after 37 DIV were recorded (Figure 7B). The LCF of the HPF was set to $3.1 \mathrm{~Hz}$ and the HCF of the LPF was set to $8.3 \mathrm{kHz}$. In our measuring system, the choice of the LCF and HFC provided the widest bandwidth in a measureable frequency range. In all 64 recording sites, the signals were successively recorded, and they were mixed signals comprised of a very rapid component (action potentials) and a slow component (FPs with lower frequencies) (Figure 7B). Therefore, the waveform of the signal shows a burst of action potentials superimposed on a slowly changing low-frequency component owing to decreasing FPs in amplitude and subsequent increasing FPs (e.g., see Ch. 51 of Figure 7C). At 37 DIV, the average noise level in these recordings was $18.3 \mu \mathrm{V}_{\mathrm{pp}}$, which is relatively larger than that $\left(<15 \mu \mathrm{V}_{\mathrm{pp}}\right)$ reported in our previous studies (Tateno and Jimbo, 1999; Tateno et al., 2005).

As described in the Materials and Methods Section, the architecture of the chip allows for the selection of an arbitrary set of electrodes for stimulation, either with individually different

Table 3 | Measured performance summary of the sensor characteristics in the chip.

\begin{tabular}{lcc}
\hline Parameter & Measured value & Unit(s) \\
\hline Process technology & $0.25 \mu \mathrm{m}$ CMOS, mixed signal & - \\
Number of channels & 64 & - \\
Chip size & $5.0 \times 5.0$ & $\mathrm{~mm}^{2}$ \\
Supply voltage & \pm 2.5 & $\mathrm{~V}$ \\
Total supply current & 563 & $\mu \mathrm{A}$ \\
Total power consumption & 1.41 & $\mathrm{~mW}$ \\
Input-referred noise & 10.1 & $\mu \mathrm{V}_{\text {rms }}$ \\
Cross-talk level & -40 & $\mathrm{~dB}$
\end{tabular}

stimulus waveforms or an identical stimulus waveform in all 64 channels. The stimulus waveforms consisted of a series of mono/bi-polar pulse trains. Figure $\mathbf{8 A}$ shows the efficiency of the on-chip reset mechanism for stimulation artifact suppression. Single bipolar pulses $(150-\mu$ s overall duration) of three different intensities $V_{\text {pulse }}(0.625,1.25$, and $2.50 \mathrm{~V})$ in an ACSF solution have been applied to one stimulation electrode (Ch. 19 in Figure 7A), while the fast reset was operational on the electrode for $V_{\text {pulse }}=0.625$ and $1.25 \mathrm{~V}$ or seemed not to be completely operational for $V_{\text {pulse }}=2.5 \mathrm{~V}$ owing to overload by voltage ranging from -2.4 to $+2.4 \mathrm{~V}$ (Figure 8Aa). With the reset mechanism, after the offset of stimulation, it took less than $3 \mathrm{~ms}$ for the recording circuitry to return close to baseline in the measuring range. In the same stimulation and recording trial, the simultaneous measurement from the stimulation and adjacent recording electrodes (Ch. 20 and 150- $\mu \mathrm{m}$ distance between the two electrodes on the substrate in Figure 7A) showed that because the reset mechanism was not operational on the adjacent recording electrode, the voltage traces on the adjacent recording electrode returned to baseline after the transient period of 20-50 ms, whose duration depended on the LCF of the HPF and the value of $V_{\text {pulse }}$ on the stimulation electrode (Figure 8Ab). However, if the voltage traces obtained from the two electrodes were compared in more detail, the slower reset mechanism was in fact operational on the stimulation electrode for $V_{\text {pulse }}=2.5$ (Figure $\mathbf{8 B b}$ ). Therefore, even for the case of $V_{\text {pulse }}=2.5$, using an off-line data analysis that included a subtraction method, action potential events were reliably detectable as shown in Figure 8Bc. The raster plot in Figure 8Bd shows spike timings (dots) derived by analyzing the data obtained from the stimulation electrode over 22 identical trials. For each trial, the first spike at around $2.5 \mathrm{~ms}$ after stimulation offset occurred with $100 \%$ reliability, while subsequent spike events were not reliable. Note that in our stimulation method as well as that reported in Hafizovic et al. (2007), a stimulation sequence always ends with stimulation of a value close to the equilibrium potential of the electrode, so that the electrode quickly returns to its equilibrium potential before the subsequent stimulation. In addition, to reduce damages to the electrodes and cells, we suggest that electrode materials with lower capacitance than Pt should be better to operate the stimulation artifact suppression mechanism properly.

\section{RECORDING AND STIMULATION IN MOUSE HIPPOCAMPAL SLICES}

In the hippocampus, long-term potentiation (LTP) is known to involve activity-dependent persistent changes in synaptic strength. LTP is a multiphasic phenomenon and current models divide it into at least three different phases: initial LTP (I-LTP), early LTP (E-LTP), and late LTP (Frey et al., 1993; Roberson et al., 1996; Sweatt, 1999; L-LTP), although an intermediate LTP phase has also been suggested (Winder et al., 1998). I-LTP represents the first stage of LTP (Kauer et al., 1988; Anwyl et al., 1989; Malenka, 1991) and is further subdivided into post-tetanic potentiation (PTP) for a persistent duration of 0.5-4 min and shortterm potentiation (STP) for a duration of 10-15 min (Schulz and Fitzgibbons, 1997). For more details regarding phases of LTP, see two recently published books (Andersen et al., 2007; Sweatt, 2009). To test our CMOS IC system in the stimulation of hippocampal slices, we focused particularly on I-LTP, because 


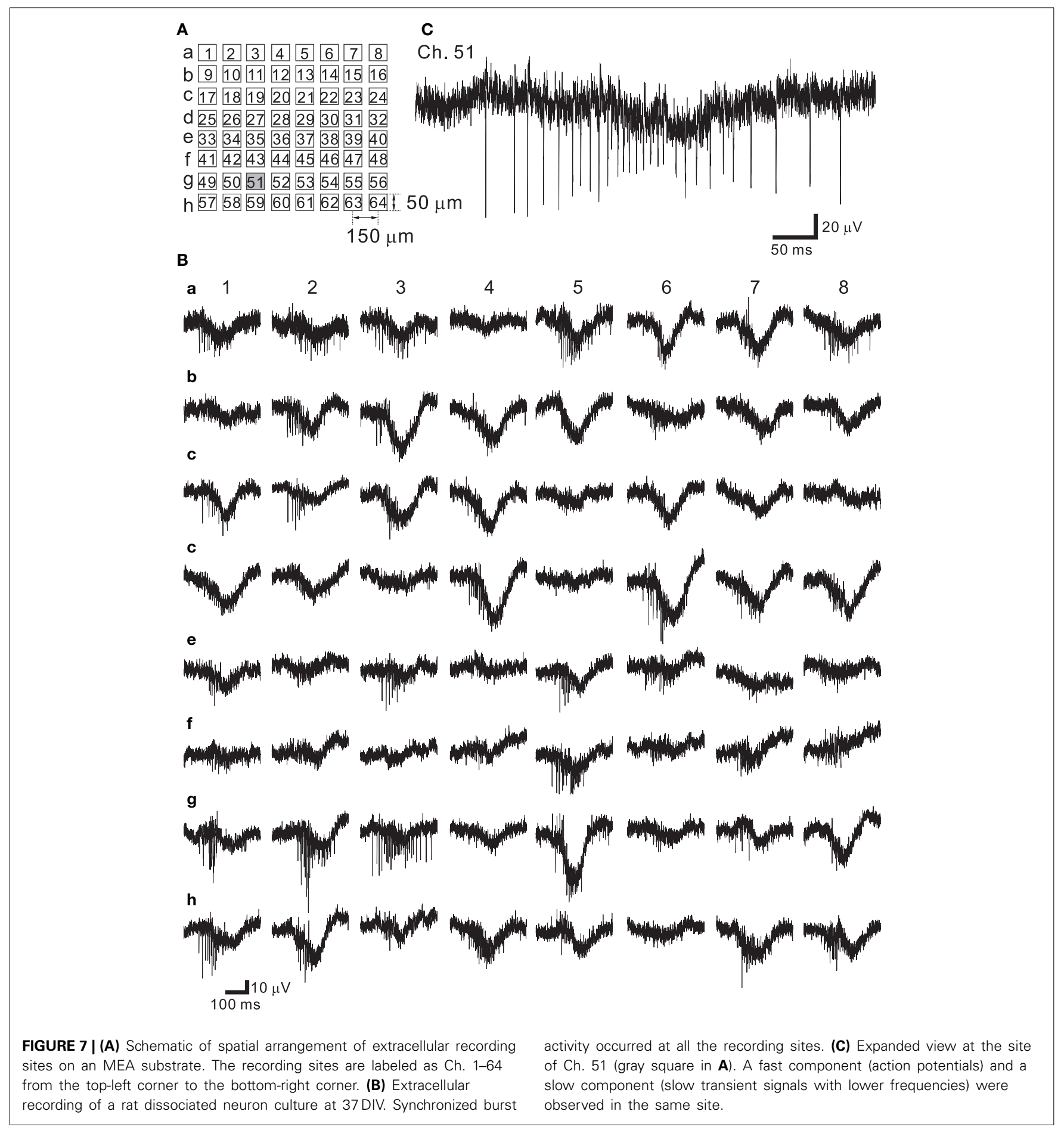

I-LTP is the first stage and the most effective for demonstrating the ability of stimulation to induce LTP during short-time in experiments.

The major input to the CA1 area of the hippocampus is from CA3 pyramidal cells via the Schaffer collaterals. Axons of CA3 pyramidal cells heavily innervate both the stratum radiatum and the stratum oriens of the CA1 area. I-LTP of Schaffer collateral fibers in hippocampal area CA1 is defined as the sustained potentiation, with a duration of at least $15 \mathrm{~min}$, that can be induced by a short burst of high-frequency stimulation (HFS). Moreover, on the basis of the time course of decremental phases, HFS can also induce two distinguishable decremental phases (PTP and STP) as stated above (Schulz and Fitzgibbons, 1997).

Figure 9A shows the placement of a slice on the MEA centered in the apical dendritic layer in the stratum radiatum of the 

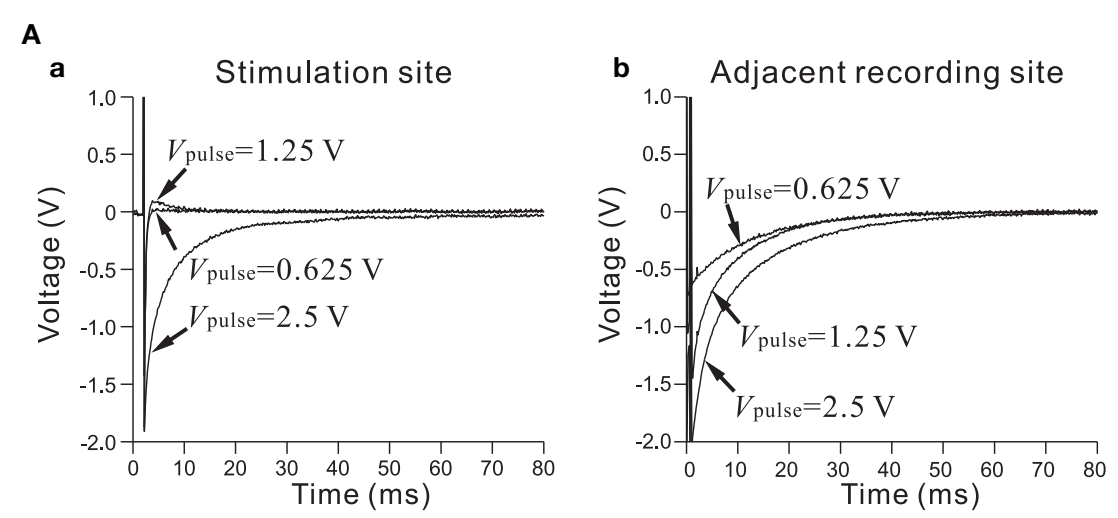

B

a

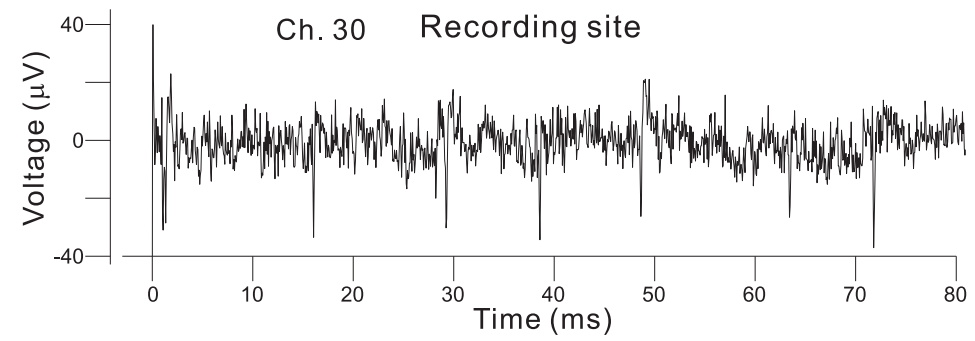

b

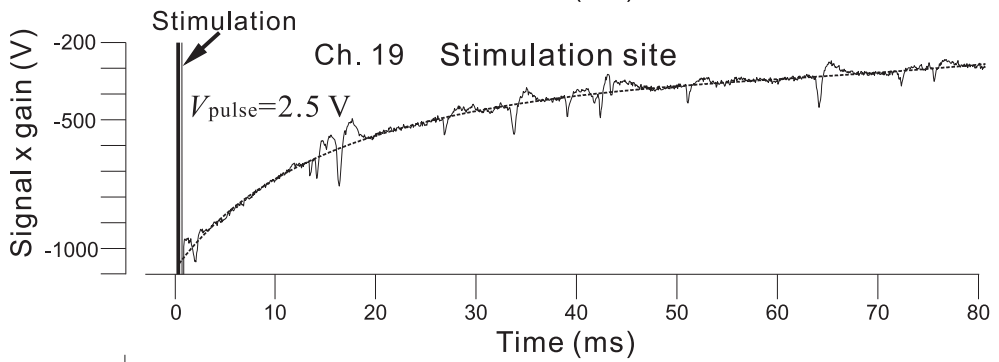

c

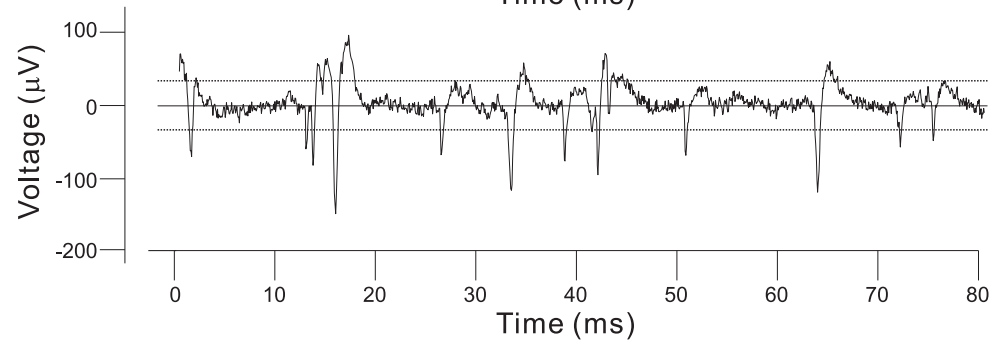

d

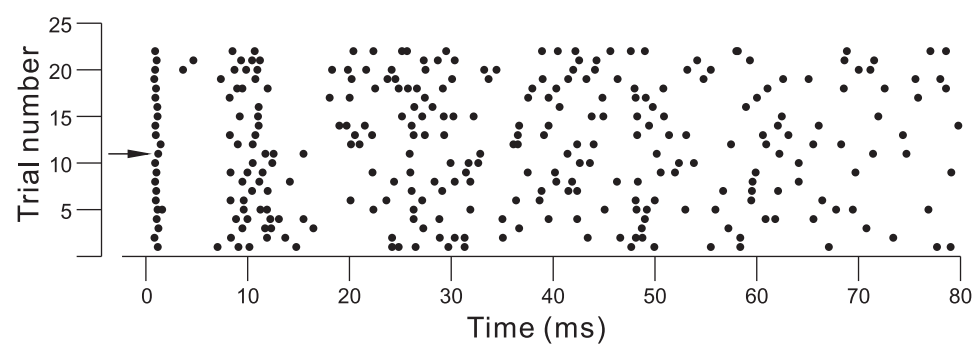

FIGURE 8 | (A) In (a), efficiency of the on-chip reset mechanism for stimulation artifact suppression on the stimulation electrode (Ch. 19 in Figure 7A). Bipolar square pulses $(150-\mu$ s overall duration) of three different $V_{\text {pulse }}$ intensities $(0.625,1.25$, and $2.50 \mathrm{~V})$ in an ACSF solution have been applied to the electrode. The individual stimulus was anodic first, cathodic second symmetrical biphasic pulses, and each pulse duration was $75-\mu$ s (i.e., $150-\mu s$ overall duration). The fast reset was operational on the electrode for
$V_{\text {pulse }}=0.625$ and 1.25 or seemed not to be completely operational for $V_{\text {pulse }}=2.5$ owing to the overload by voltage ranging from -2.4 to $+2.4 \mathrm{~V}$. With the reset mechanism, after the offset of stimulation, it takes less than $3 \mathrm{~ms}$ for the recording circuitry to return close to baseline in the measuring range. In (b), on the adjacent recording electrodes (Ch. 20 and 150- $\mu \mathrm{m}$ distance between the two electrodes on the substrate in Figure 7A), 


\section{FIGURE 8 | Continued}

because the reset mechanism was not operational on the recording electrode, the voltage traces returned to baseline after a transient period of $20-50 \mathrm{~ms}$. Although the fast reset mechanism was not operational on the adjacent recording sites in the particular experiment, it can be possible. (B) In (a), spike events in recording signals from rat cultured cortical neurons at $37 \mathrm{DIV}$ are detectable on a recording electrode (Ch. 30). With this recording filter setting, the gain, LCF, and HCF were $39.4 \mathrm{~dB}$, $28.6 \mathrm{~Hz}$, and $8.3 \mathrm{kHz}$, respectively. In (b), for $V_{\text {pulse }}=2.5 \mathrm{~V}$, a transient voltage trace to baseline was obtained from the recording on the stimulation electrode (Ch. 19 in Figure 7A). The transient waveform was approximately fitted by a 5-th order polynomial as a function of time, as indicated by a dotted curve. In (c), a waveform including action potentials is shown after subtracting the polynomial function from the trace in (b). Comparison of the two waveforms shown in (a) and (c) indicates that action potentials in (c) are large enough to detect the event timings. For spike detection threshold, two dotted lines indicate values three times the standard deviation of the baseline noise. In (d), a raster plot shows spike timings (dots) derived from the analysis of data obtained from the stimulation electrode for 22 identical trials. For each trial, the first spike at around $2.5 \mathrm{~ms}$ after the stimulation offset occurred with $100 \%$ reliability, while subsequent spike events were not reliable.
CA1. In this study, for orthodromic stimulation, a stimulating site was usually selected in the stratum radiatum; for example, the electrode labeled as Ch. 37 was the stimulation site in this particular experiment. Extracellular field population excitatory postsynaptic potentials (EPSPs) in all sites, excluding the stimulation site, are shown in Figure 9B. With this placement of the slice on the array, population spikes were recorded on some upper electrodes in the cell body layer of the stratum pyramidale (e.g., Ch. 21 in Figure 9Ca), and monophasic population EPSPs were recorded in the apical dendritic layer of the stratum radiatum (Ch. 40 in the Figure $9 \mathbf{C b}$ ). In addition to a usual LTP experiment, a single bipolar pulse $( \pm 1.25$ or $\pm 2.50 \mathrm{~V})$ was applied per trial at the beginning of a series of three sessions, and several trials were performed as a control session. Next, at time $t=0$ after the control session, HFS, referred to as the TS, was applied once at $125 \mathrm{~Hz}$ with an intensity of $2.5 \mathrm{~V}$ for a duration of $0.8 \mathrm{~s}$ (recording site Ch. 21 in Figure 10A) at a single site (Ch. 37). Then, single bipolar pulses were repetitively applied as a test session for over 15 min with the same stimulation parameter set as used in the control session. Examples of population spikes from the series of sessions are shown in Figure 10B before and after TS (i.e., at time points of $t=-5,-1,+1$, and $+16 \mathrm{~min})$. One minute after TS $(t=+1 \mathrm{~min})$, amplitudes of the first positive and negative peaks $\left(\mathrm{P}_{1}\right.$ and $\mathrm{N}_{1}$ in Figure 10B) increased, but gradually decreased as time passed. For example, the $\mathrm{N}_{1}$ amplitude at $16 \mathrm{~min}$ after TS $(t=+16 \mathrm{~min})$ was $27 \%$ smaller than that at $1 \mathrm{~min}$ after TS $(t=+1 \mathrm{~min})$, although the amplitude was still larger than those of the control level at 1 and $5 \mathrm{~min}$ before TS $(t=-5$ and $-1 \mathrm{~min})$. To quantify the peak intensities of both the population spikes and EPSPs at multiple sites during a series of trials, voltage differences between $\mathrm{P}_{1}$ and $\mathrm{N}_{1}$ were calculated (Figure 10C). The results from over 20 sites indicated that the intensities of the population spikes and EPSPs after TS were increased by over $50 \%$ for three slices $(n=3)$. Similarly, on the basis of pre-tetanic negative peak amplitude $\left(\mathrm{N}_{1}(t=-1)\right)$, negative peak voltage differences (i.e., $\mathrm{N}_{1}(t=+5)-\mathrm{N}_{1}(t=-1)$ ) between two time points were calculated (Figure 10D). At 26 sites, the $\mathrm{N}_{1}$ voltage difference showed a significant change $(p<0.01)$. In short summary, these results indicate that our CMOS IC-based multichannel system is capable of inducing I-LTP in mouse hippocampal slices. In particular, the voltage stimulus intensities in these experiments were confirmed to be large enough to induce I-LTP.

\section{RECORDING AND STIMULATION IN MOUSE BRAIN SLICES INCLUDING THE AUDITORY CORTEX}

In general, sensory information from the thalamus enters the sensory cortex by way of thalamocortical synapses to neurons in cortical layer 4 (L4), and these L4 neurons then transmit the information to neurons in L2 or L3 and then to L5 (for review of the auditory cortex, see Winer et al., 2005; Schreiner and Winer, 2007). Happel et al. analyzed cortical laminar profiles of tone-evoked current source density (CSD) in response to acoustic stimulation of the in vivo auditory cortex with pure tones, and revealed characteristic spatiotemporal pattern changes between current source and sink in the CSD profiles (Happel et al., 2010). Using our CMOS IC system and in vitro mouse preparations including the auditory cortex, we expected to demonstrate similar CSD profiles observed in the auditory cortex in vivo. Evoked FPs in cortical slices were recorded using the 64 MEA channels when a bipolar short pulse was applied to one of the 64 stimulation and recording electrode sites. In addition, to mimic the peripheral auditory system and to trigger the stimulation and recording of the CMOS IC system, a piezoelectric acoustic sensor on a custom-made signal processing board (SPB) was used (for more details, see Materials and Methods and Tateno et al., 2013), as shown in Figure 11A. The SPB transforms acoustic sound (e.g., a tone burst with a specific frequency component in Figure 11Ba) into an electric signal (Figure 11Bb) via the piezoelectric sensor, and the signal is substantially rectified and integrated (Figure 11Bc). Once the integrated signal is increased over a fixed threshold, a trigger signal to start stimulation and recording is applied to the CMOS IC system (Figure 11Bd and the timing chart in Figure $\mathbf{3 A b}$ ). A mouse coronal slice including the primary auditory cortex (A1) overlying an MEA is shown in Figure 11C. When the top row of the electrodes was placed on the surface layer (i.e., L1), the fourth row from the top was typically located in L4 or the border between L3 and L4. A short bipolar pulse was then applied to an electrode on the fourth row (e.g., Ch. 29 in Figure 11C); the observed profiles of FPs on two columns (c4 and c6 in Figure 11C) of electrodes are shown in Figure 11D. Around $5 \mathrm{~ms}$ after the stimulation offset, the maximum amplitude of the FP trace at the electrodes occurred at the sites (Chs. 28 and 30) adjacent to the stimulation site (Ch. 29), and the activity propagated to the superficial and deeper layers. The propagated negative peak was most increased at the electrode in L3 (the third row from the top), although the stimulation electrode was located on the fourth row from the top. Because similar 

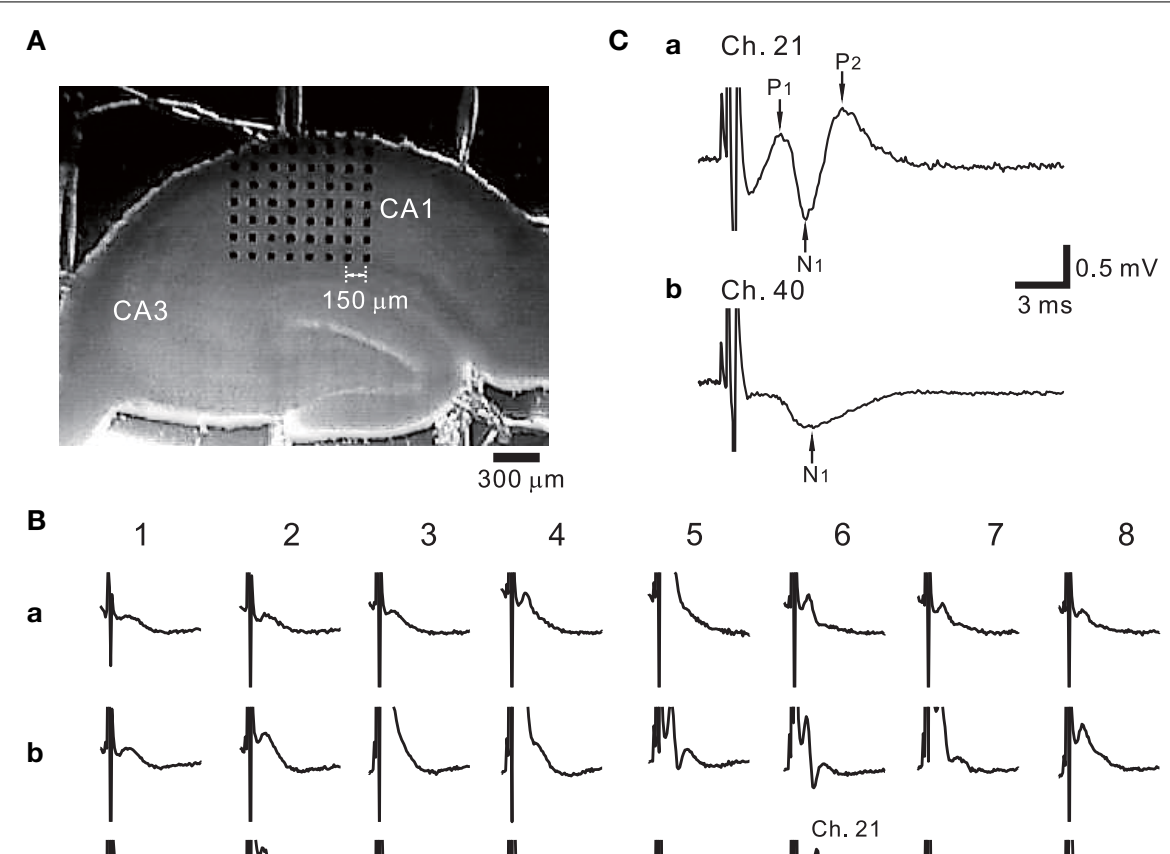

c
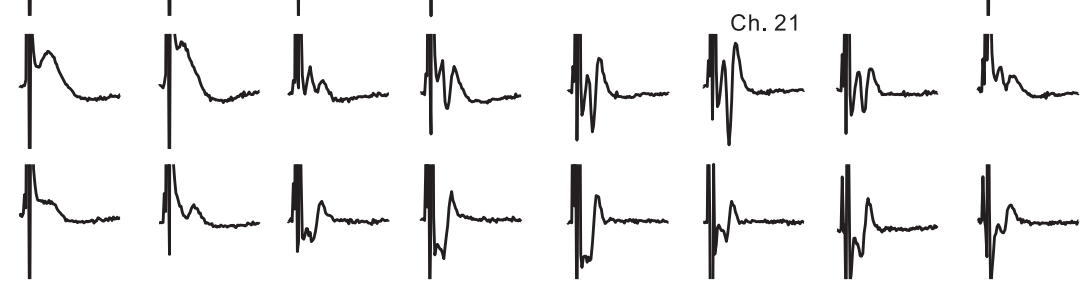

d
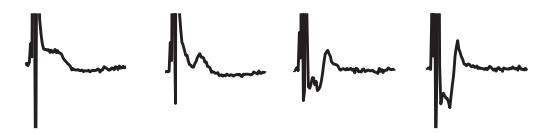

e $\quad \|$ mm

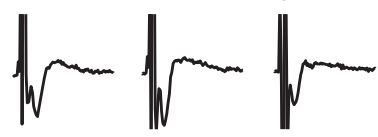

Stimulation
site:
Ch. 37
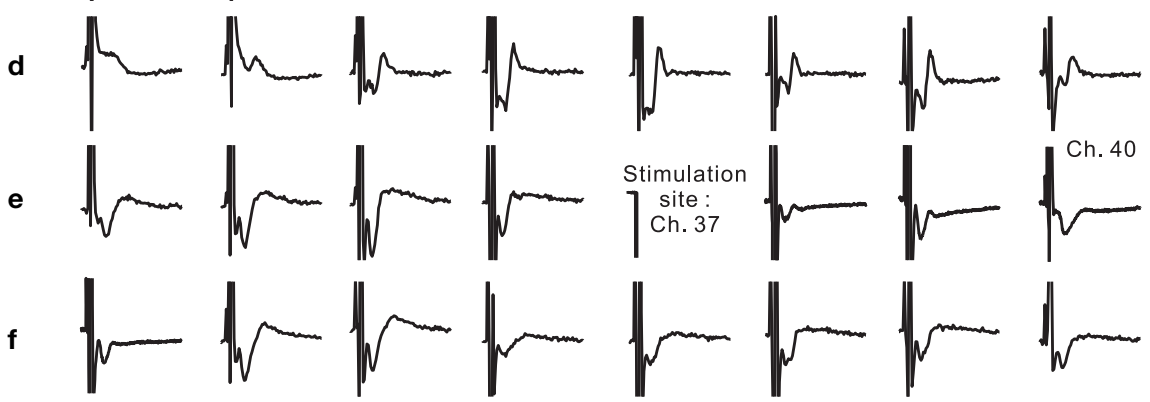

g
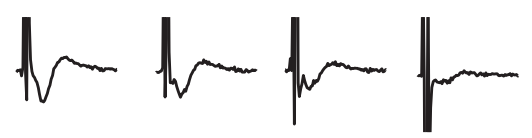

h
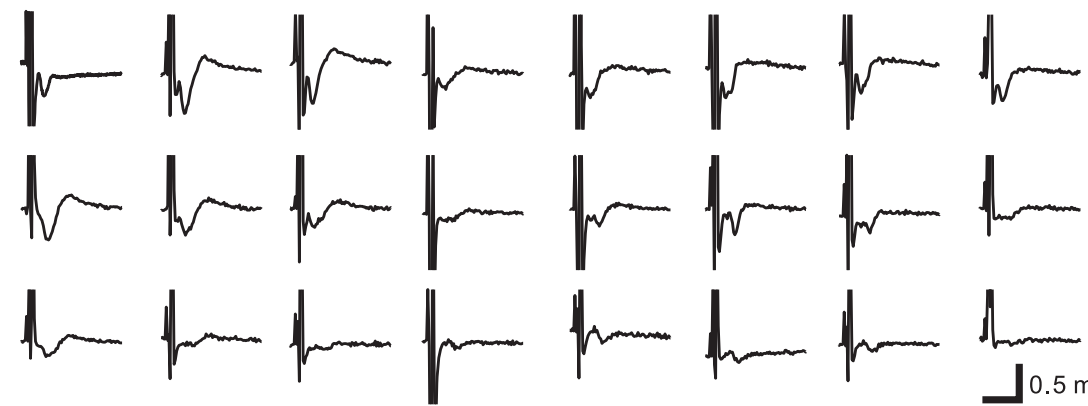

FIGURE 9 | (A) One example of hippocampal slice placement on the multielectrode array (MEA) centered in the apical dendritic layer in the stratum radiatum of the CA1. The MEA probe consisted of an array of 64 planar microelectrodes, each $50 \times 50 \mu \mathrm{m}$, arranged in an $8 \times 8$ pattern with a $150-\mu \mathrm{m}$ interpolar distance. (B) Extracellular recording of field potentials from a mouse slice including the hippocampus. In response to a short bipolar stimulus $( \pm 2.5 \mathrm{~V})$ at $\mathrm{Ch}$. 37 , field population spikes and

FP profiles were always observed ( $n=10$ slices) in the same slice configuration on MEAs, the corresponding CSD profiles shows typical source-sink-source triplet patterns (Figure 11D). Hence, the characteristic CSD patterns were reminiscent of the CSD patterns obtained in in vivo responses evoked by a pure tone stimulus (Happel et al., 2010). The above result implies that in vivo microstimulation of L4 neurons of a specific column in a tonotopic map of A1 can provide similar CSD patterns during intrinsic sound processing in $\mathrm{Al}$ in response to pure-tone stimuli.

\section{DISCUSSION}

In this section, we briefly summarize standard and advanced techniques used in designing a CMOS IC-based multichannel system for recording and stimulation of neural networks (for 
A a Ch. 21, Tetanic stimulation, $125 \mathrm{~Hz}$

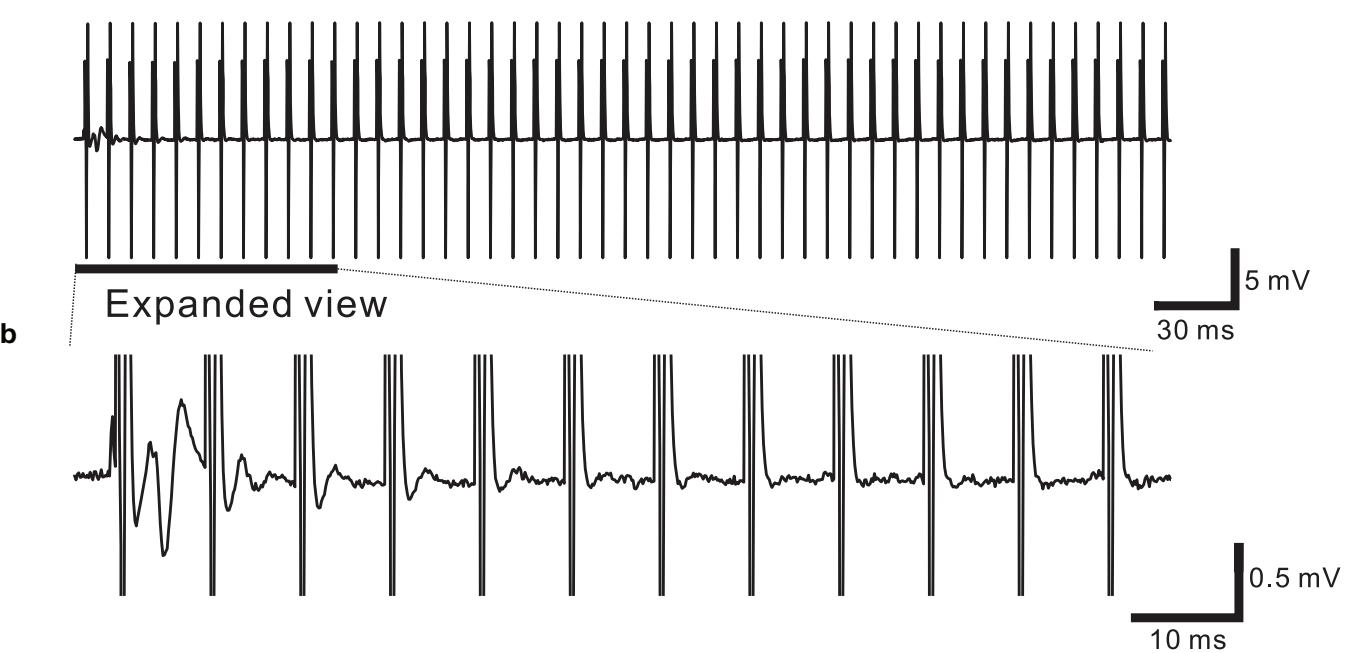

B Ch. 21

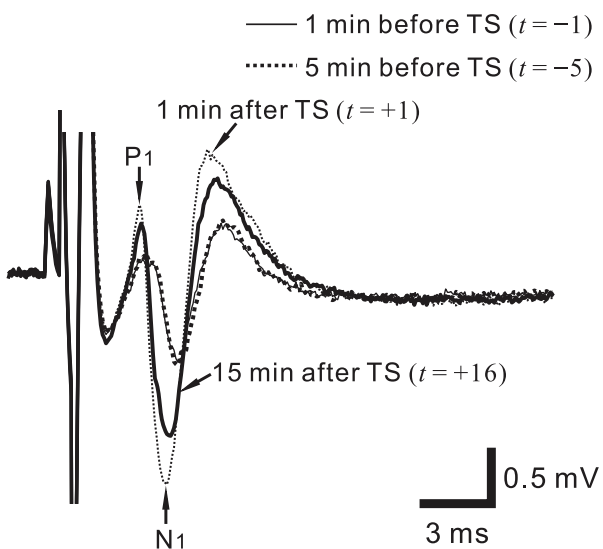

c Chs. 1-32

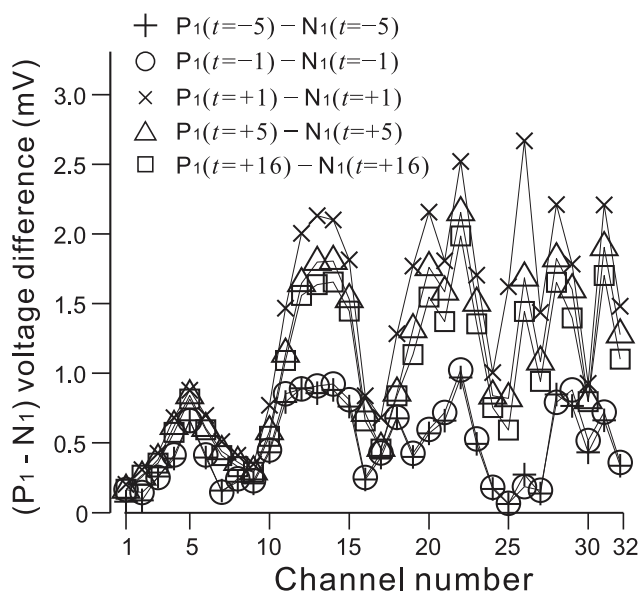

D Chs. 1-64

Channel number

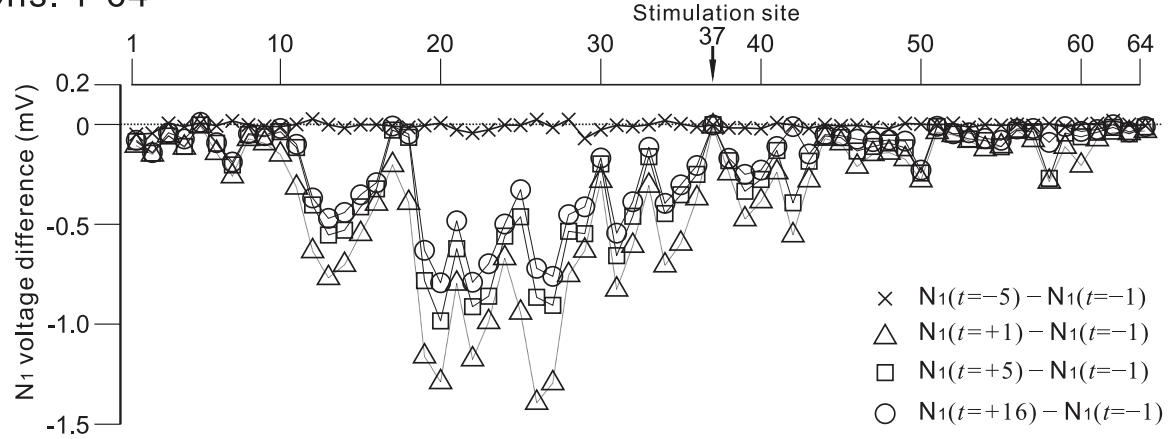

FIGURE 10 | (A) In (a), a typical example of recorded field potentials in response to a high-frequency stimulation (HFS), referred to as the tetanic stimulation (TS), at a recording site, Ch. 21 (cf. Figures 7A, 9A). The TS applied at a stimulation site (Ch. 37) consists of 100 repetitions of identical short bipolar pulses with an intensity of $2.5 \mathrm{~V}$ at $125 \mathrm{~Hz}$ for a duration of $0.8 \mathrm{~s}$. In (b), in response to the same TS shown in (a), the expanded view at the recording site is illustrated for the time interval that includes the first 12 pulses. The evoked responses of the field potential were gradually reduced in amplitude as the pulses were repeatedly applied. (B) From the time when the TS was applied ( $t=0 \mathrm{~min}$ ), examples of field population spikes at four time points: 5 min before the TS ( $t=-5$, thick dotted curve), 1 min before the TS ( $t=-1$, thin curve), 1 min after the TS ( $t=+1$, thin dotted curve), and $16 \mathrm{~min}$ after the TS ( $t=+16$, thick curve). After the stimulation offset, the first positive and negative peaks are labeled by $P_{1}$ and $N_{1}$, respectively. (C) Voltage differences between $\mathrm{P}_{1}$ and $\mathrm{N}_{1}$ at Chs. 1-32 for each time point ( $t=-5,-1,+1,+5$, and $+16 \mathrm{~min}$ ) to quantify peak intensities of the population spikes and EPSPs at multiple sites during a series of trials. (D) On the basis of pre-tetanic negative peak amplitude $\left(N_{1}(t=-1)\right)$, in all 64 sites, negative peak voltage differences between two time points are calculated; that is, $N_{1}(t=s)-N_{1}(t=-1)$, where $s=-5,+1,+5$, and $+16 \mathrm{~min}$. 

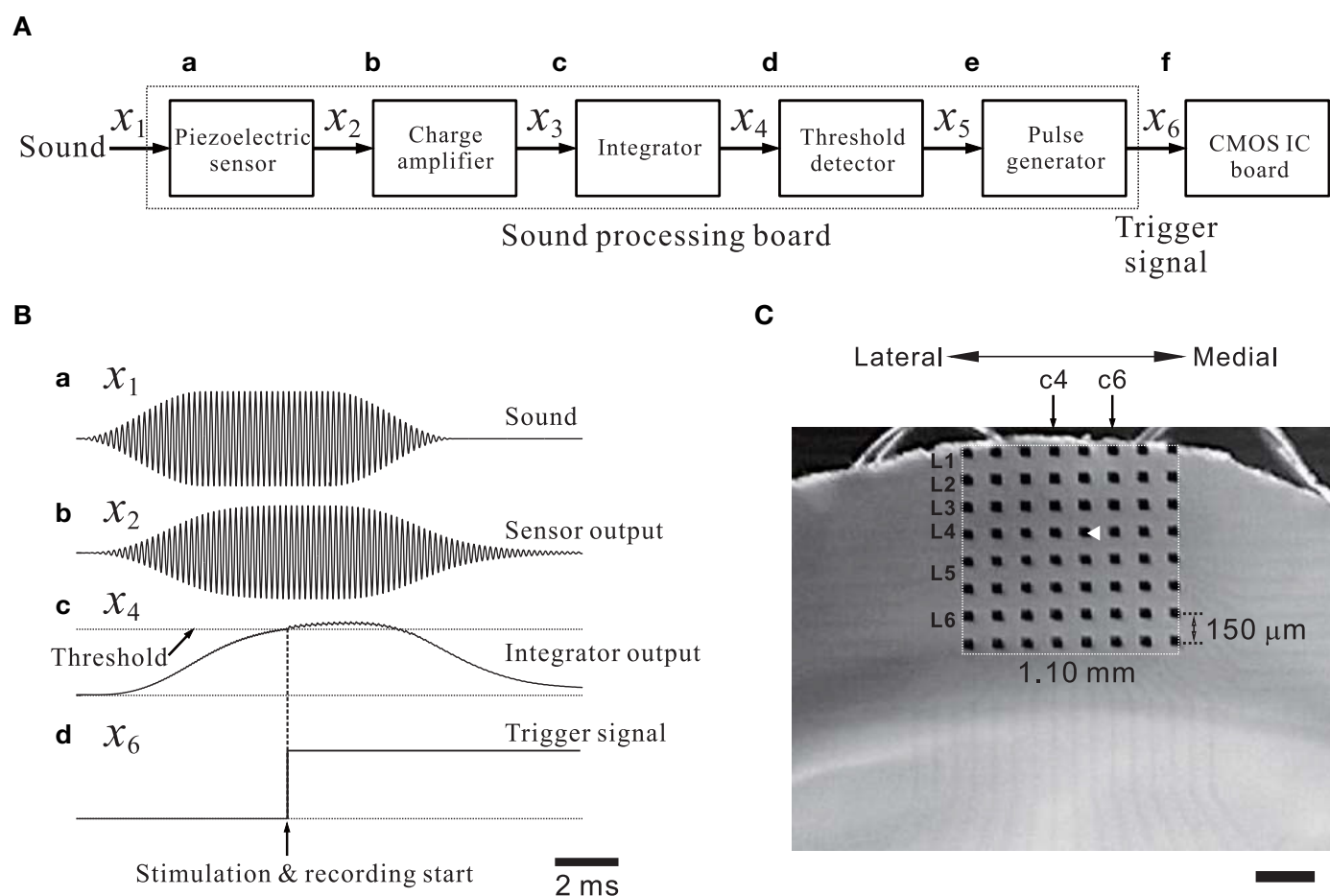

Sound processing board

Trigger

signal

C

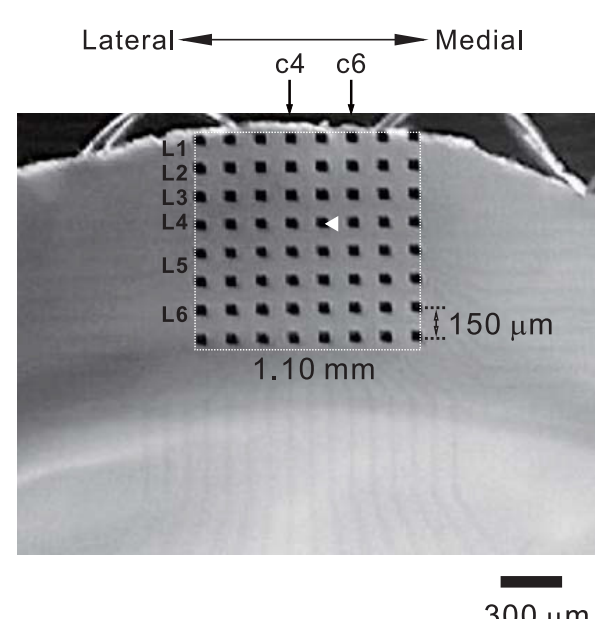

D

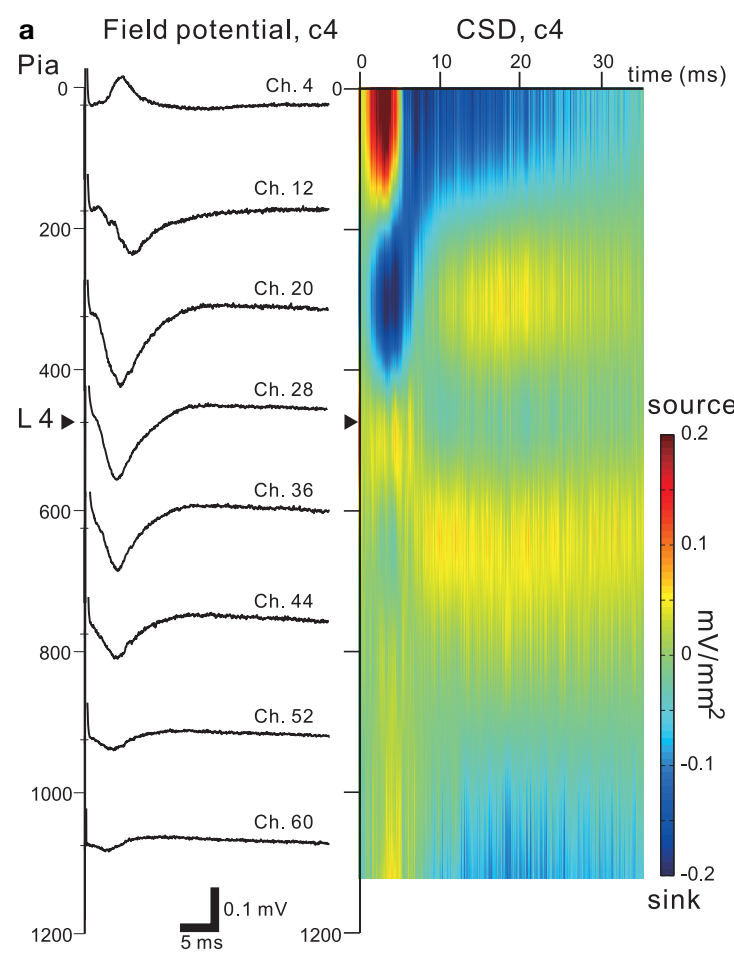

b Field potential, $c 6$

$300 \mu \mathrm{m}$

FIGURE 11 | (A) A simple block diagram of the acoustic sound processing board (SPB) for mimicking the mammalian peripheral auditory system. A pulse generator to trigger the stimulation and recording processes of the CMOS IC system (CMOS IC board) was incorporated using the output signal of the SPB. Signals on the SPB are processed from left (sound) to right (CMOS IC board). The variables above arrows between two blocks indicate the corresponding signals transmitted from the left to the right block. (B) In

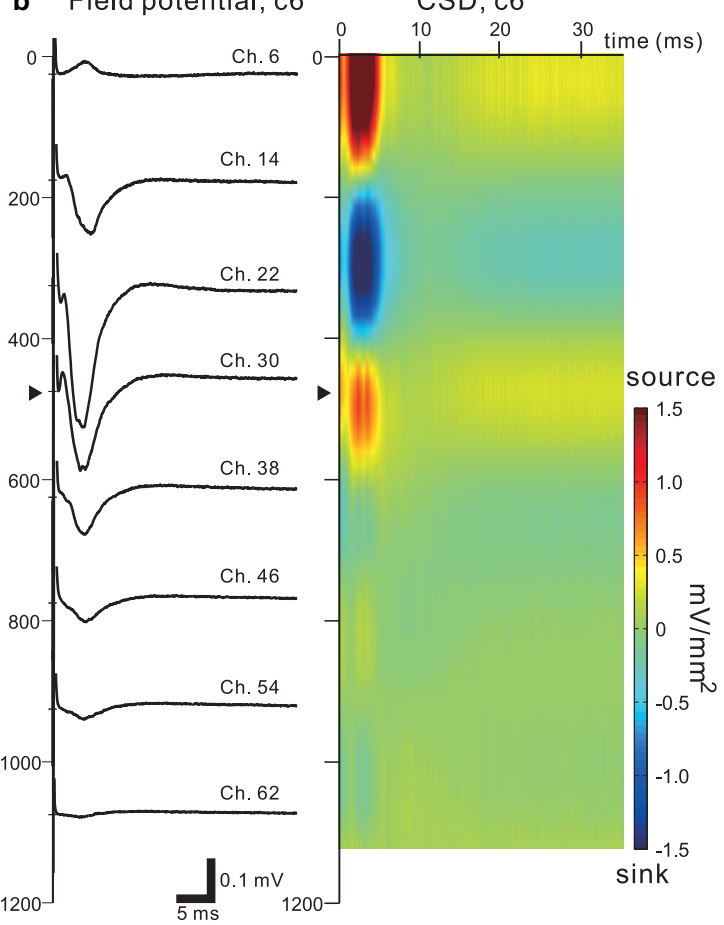

(a), a transmitted acoustic signal $x_{1}$ from a speaker to the sensor is shown. The sound level was $75 \mathrm{~dB}$ SPL. Similarly, in (b-d), the waveforms indicate a sensor-output signal $x_{2}$, an integrator signal $x_{4}$, and a trigger signal $x_{6}$ applied to the CMOS IC board, respectively. The threshold level of the threshold detector was $1.4 \mathrm{~V}$, and the output voltage of the pulse generator was $2.5 \mathrm{~V}$. For more details, see Tateno et al. (2013). (C) A mouse coronal slice including (Continued) 


\section{FIGURE 11 | Continued}

the primary auditory cortex overlaid on the MEA substrate. The MEA probe is the same as shown Figure 9A. The covered area on the MEA probe of the electrodes is $1.10 \times 1.10 \mathrm{~mm}^{2}$. The position of the laminar cortical structure is approximately indicated by labels illustrated as layer 1 (L1) to L6. The stimulation site (Ch. 29) was indicated as a white triangle. The individual stimulus was anodic first, cathodic second symmetrical biphasic rectangular

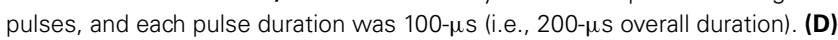
Profiles of FPs (left) and CSD (right) are shown for two columns (c4 and c6) of eight electrodes on the left and right sides of the stimulation site (Ch. 29). comprehensive review, see Gosselin, 2011; Demosthenous, 2014) and compare them with the specific system we designed here. Next, we discuss the electrophysiological applications of our system to the three neural preparations in vitro and a future plan for a CMOS IC-based prosthesis for the auditory cortex in vivo.

\section{SYSTEM-LEVEL ARCHITECTURE}

Front-end neural recording interfaces for multi-electrode systems are typically based on two architectures; analog and digital timedivision multiplexing (TDM) (Rodriguez-Perez et al., 2013). In the analog TDM architecture (Hafizovic et al., 2007; Shahrokhi et al., 2010), the analog front-end circuits that amplify and filter the neural signal acquired from each of the electrodes are grouped in an array of channels. Each of these channels comprises an LNA and a bandpass filter, followed by a programmable gain amplifier to maximize the output swing. The analog outputs from all the channels are then multiplexed in the time domain, and they are subsequently converted into digital words by an ADC. The generated time-multiplexed data frames can be either digitally processed to compress them, or sent directly to the output as raw data. In this study, we also employed the analog TDM architecture, and TDM data were stored in a PC through the FPGA card. The analog TDM architecture is likely to have an advantage for an off-chip controller using amplified neural signals in real time for closed-loop control and stimulation (Rolston et al., 2010), although such a controller would be implemented in an on-chip design.

In contrast, in the digital TDM architecture (Gosselin et al., 2009; Seung Bae et al., 2010) the ADC is not shared between the analog channel outputs; rather, an ADC is embedded in each channel. Then a common digital processor manages the digitized signals from the channels, classifies (or reduces) the data, and sends it to the output. In general, this solution requires a larger silicon area than the approach used by the analog TDM architecture. However, the digital TDM architecture has advantages in power consumption owing to the much lower sampling rate requirement at the digitization stage. In addition, the digital TDM architecture has the benefit of being easily scalable by replicating the channels. A very compact circuit implementation for the topology in the digital TDM architecture is proposed in some studies (e.g., Harrison and Charles, 2003), requiring a very small silicon area per channel with standard CMOS process technology. Exploring this implementation could be one of the future directions of our work.

\section{LOW-NOISE AMPLIFIERS}

In recording neural activity with planar multielectrodes and penetrating microelectrodes such as the Utah Electrode Array (Rousche and Normann, 1992; Nordhausen et al., 1996) or the Michigan penetrating probes (Wise et al., 1970; Wise, 1986), the recorded neural action potentials often have amplitudes of only a few tens of microvolts, with most energy concentrated between $100 \mathrm{~Hz}$ and $6 \mathrm{kHz}$ (Najafi, 1994). Hence, circuits for neural signal amplification must have low noise performance. In addition, front-end neural amplifiers are required to reject electrode offsets or common-mode interference. In the design of such neural amplifiers, two major methods have been used: clock-based and continuous-time techniques (Gosselin, 2011; Demosthenous, 2014). The noise in CMOS transistors is usually dominated by flicker $(1 / f)$ noise up to relatively high frequencies of the order of several tens of $\mathrm{kHz}$ (Nemirovsky et al., 2001). Therefore, various clock-based techniques have been developed to reduce the effects of $1 / f$ noise, and the noise reduction technique is based on switched biasing (Klumperink et al., 2000; Min et al., 2012), chopper modulation (Enz et al., 1987; Denison et al., 2007), and autozeroing (Enz and Temes, 1996; Masui et al., 2007). However, the clock-based techniques described above require a clock generation circuit, and thus suffer from potential problems associated with high-frequency interference and clock feedthrough. In addition, high-frequency switching circuits can increase the complexity and power consumption of the design. Hence, in this study, we did not adopt the clock-based technique.

In contrast, continuous-time techniques have been extensively used in the design of neural amplifiers. The conventional circuit in the continuous-time technique is the AC-coupled operational OTA-based neural amplifier with capacitive feedback (Harrison and Charles, 2003), and it is this circuit that we used in this study. As shown in Figure 2A, the circuit is built around a single-stage OTA with CMOS technology. In addition, as described in the Materials and Methods Section, the ratio of capacitors $C_{1}$ and $\mathrm{C}_{2}$ in Figure 2A sets the mid-band gain of the bandpass response (Figure 5A). The input is capacitively coupled through $C_{1}$, so that any DC offset from the electrode-tissue interface is removed. To achieve this, $C_{1}$ should be made much smaller than the electrode impedance to minimize signal attenuation (cf. Table 1). Hence, we designed an array of capacitors $C_{1}$ and realized selectable mid-band gains with either one capacitor or a combination of several (Figure 2B). Moreover, transistors implement MOS pseudoresistors $R_{\mathrm{f}}$ with an extremely large incremental resistance $\left(\approx 10^{10-12} \Omega\right.$, cf. Table 1$)$. This allows the LCF of the input HPFs of the AC-coupled stage to be set to a smaller value than several hertz or millihertz. The LCF is set by the product of $C_{2}$ and the MOS pseudoresistor $R_{\mathrm{f}}$ (Figure 2A), although small changes in $R_{\mathrm{f}}$ cause variance of LCFs in LNAs. Additionally, in our system, the smallest LCF of HPF is around $3 \mathrm{~Hz}$. This limitation prevents the system from properly recording delta frequencies and restricts its applicability to in vivo studies targeting at lower rhythms. The limitation is likely to be owing to leakage current of the MOS pseudoresistor. Possible other strategies to overcome this limitation could be to increase values of capacitance $C_{2}$ and/or to 
change the MOS transistor type (i.e., replacement of PMOS transistors with NMOS transistors). The HCF is a function of the load capacitance $\left(C_{3}\right.$ in Figure $\left.2 \mathrm{~B}\right)$, the OTA transconductance $\left(g_{\mathrm{m}}\right)$, and the mid-band gain $\left(C_{1} / C_{2}\right)$. Therefore, to adjust the LCFs and HCFs to appropriate values for target signals, we designed arrays of $R_{\mathrm{f}}$ and $C_{3}$ (Figure $2 \mathrm{~B}$ ). As a result, we could successfully record FP signals and single spikes by changing the LCFs and HCFs of the LNA and adjusting the frequency band.

\section{ARTIFACT CANCELATION CIRCUIT AND ARTIFACT SUPPRESSION}

To study connectivity of neuronal networks and evoked responses of neural projections, multi-site electrical stimulation is necessary (Wagenaar and Potter, 2004; Heer et al., 2006; Whitson et al., 2006). For example, Wise et al. developed a 3D-electrode array that had separate stimulation and recording probes, and each of the probes featured 64 sites and 8 channels in in vivo applications (Wise et al., 2004). Olsson et al. developed thin-film MEAs with integrated circuitry for extracellular neural recording in behaving animals, as well as an eight-site probe for simultaneous neural recording and stimulation (Olsson et al., 2005). In addition, in the context of bidirectional interaction with neural networks, recording neural signals shortly after the stimulation pulse has been applied usually results in stimulation artifacts that mask the neural signals. To reduce these artifacts, Jimbo et al. (2003) for the first time developed an artifact cancelation circuit and incorporated it into a multichannel recording system. In particular, Jimbo et al. presented an MEA-based system where electrodes can be switched between recording and stimulation, using the fast stimulation reset mechanism to record neural signals on stimulating electrodes $3 \mathrm{~ms}$ after stimulation (Jimbo et al., 2003). Many CMOS IC-based artifact suppression systems have been developed in recent several studies (Heer et al., 2006; Hafizovic et al., 2007; Hottowy et al., 2008).

In such systems, the stimulation protocol should generally meet two requirements (Jimbo et al., 2003). Firstly, to avoid overloading the amplifier, the input of the recording amplifier has to be disconnected from the electrode during the stimulation period; i.e., there is the capability to perform fast switching from stimulation to recording and vice versa, even on the same electrode (Hafizovic et al., 2007). In the next stage, the amplifier should be connected to a constant potential equal to the electrode DC offset. Secondly, the residual voltage on the electrode should be discharged after the stimulation pulse as quickly as possible. The residual voltage on the electrode after stimulation can generally be minimized by precise balancing of bipolar stimulation pulses. In practice, it is rather difficult to reduce residual voltages to zero, so one must deal with values on the order of several millivolts. Taking into account these considerations, we have developed the circuit shown in Figure 3. In our MEA system, the minimum transition time from the recording mode to the stimulation mode is about $63 \mu \mathrm{s}$. Furthermore, it takes about $1.2 \mathrm{~ms}$ in addition to the switching time to sense the evoked neural spikes because the capacitance at the electrode/electrolyte interface discharges toward the initial state (Jimbo et al., 2003). Thus, $1.3 \mathrm{~ms}$ after stimulus offset is the minimum latency for recording neural spikes. In combination with off-line data analysis, we can successfully detect spikes within several milliseconds after offset of stimulation (cf. Figure 8) in dissociated cultured neurons, even if the applied voltage intensity is relatively large (e.g., $2.5 \mathrm{~V})$. In contrast, new approaches are needed to record evoked potentials on the stimulation electrodes in the acute slice preparations, because they retuned more slowly to the initial state than those in the cultures (Buitenweg et al., 2000; Braun and Fromherz, 2004; Franks et al., 2005).

\section{RECORDING AND STIMULATION OF THREE PREPARATIONS IN VITRO AS A TEST BENCH}

Cultured cortical neurons were previously found to produce synchronized bursts during their early development (Kamioka et al., 1996; Watanabe et al., 1996). The observed signals from cultured neurons on an MEA substrate were (i) single spikes, (ii) bursts, and (iii) slowly changing FPs with time constants of several hundreds of milliseconds. These signals, each with different frequency components, are all recorded by our CMOS IC-based multichannel system (Figure 7). Although we have not illustrated all examples here, by selecting appropriate LCFs and HCFs we can use this system to filter target neural signals.

The hippocampus plays an indispensable role in many important brain functions, including memory formation and spatial navigation. Also, it is very well suited for slice studies owing to the planar organization of projections among hippocampal subfields. Therefore, a number of researchers have pursued the study of the hippocampus with MEAs (Novak and Wheeler, 1988; Egert et al., 1998; Shimono et al., 2000; Chong et al., 2011). Although these experiments could be performed with traditional instrumentation and a conventional MEA-based multichannel system, we intended to extend the range of such recording systems to our CMOS IC-based multichannel system. Because there is increased throughput owing to the ability to stimulate and record from multiple sites, the different responses expected in the various regions act as within-slice controls that help validate the viability of the slice and the experimental conditions. In this study, by applying a short burst of HFS to Schaffer collateral fibers in hippocampal area CA1, we could induce sustained postsynaptic potentiation with a duration of over $15 \mathrm{~min}$. These results indicate that the stimulation intensity of our system was large enough to induce sustained changes in mouse hippocampal slices.

To test the validity of our system, we applied voltage stimulation to acute mouse slices including the auditory cortex. The stimulation intensities were large enough to evoke large local FPs ranging from several hundred microvolts to several millivolts (Figure 11), so that the obtained responses were suitable for further analysis, for example CSD analysis. In this study, CSD analysis results were reminiscent of source-sink-source triplet CSD profiles from in vivo recording in the auditory cortex in response to an acoustic sound stimulus. In addition, to trigger electrical stimulation and recording, we connected the acoustic piezoelectric sensor to the CMOS IC-based multichannel system. The entire system structure we developed can serve as the basis for a neural prosthesis for the auditory cortex in vivo. To realize this goal in the near future, the method of converting sound into a stimulation pattern in the brain tissue in vivo is the next important target of our study. 
An additional related challenge is to design high-resolution CMOS IC-based microelectrode systems that can provide bidirectional access to several individual cortical neurons in vivo via electrical stimulation and neural recording capabilities in the same device. Such systems will make it possible to interact with groups of neurons in a closed loop (Rolston et al., 2010); i.e., (i) to make use of electrical stimulation in conjunction with neural recording to activate specific neural networks, (ii) to obtain instantaneous feedback information on their status, (iii) and to electrically modify the activity of the networks as well as the device system parameters. Developing such CMOS IC-based bidirectional neural interfaces will have a profound impact on experimental neuroscience and on the implementation of new assistive technologies to treat chronic diseases and neural disorders, including tinnitus, epilepsy, and Parkinson disease.

\section{CONCLUSIONS}

We describe an ASIC system designed for multisite electrical stimulation of neural tissue using MEAs. Our design is intended for applications in systems requiring simultaneous stimulation and recording of signals from various types of neural tissue, both in vitro and in vivo. The developed ASIC comprises 64 independent stimulation channels, any subset of which may be stimulated, and can generate almost arbitrarily defined bipolar voltage pulses below an amplitude of $2.5 \mathrm{~V}$ with 5-bit resolution. Each channel is also equipped with a stimulation artifact suppressor controlled in real time, which reduces the dead time of the system after each stimulation pulse. The system we developed can be used for conventional electrophysiological experiments in vitro, and the technology is also a foundation of future auditory neural prostheses applied to the auditory CNS.

\section{ACKNOWLEDGMENTS}

This work was supported by the Funding Program for Next Generation World-Leading Researchers (NEXT program) and by a research grant from the Okawa Foundation. We appreciate Professor Atsushi Iwata and Mr. Masatoshi Ono for their valuable comments.

\section{SUPPLEMENTARY MATERIAL}

The Supplementary Material for this article can be found online at: http://www.frontiersin.org/journal/10.3389/fneng. 2014.00039/abstract

\section{REFERENCES}

Andersen, P., Morris, R., Amaral, D., Bliss, T., and O'Keefe, J. (2007). The Hippocampus Book. New York, NY: OUP.

Anwyl, R., Mulkeen, D., and Rowan, M. J. (1989). The role of N-methyl-D-aspartate receptors in the generation of short-term potentiation in the rat hippocampus. Brain Res. 503, 148-151. doi: 10.1016/0006-8993(89)91716-2

Berdondini, L., Imfeld, K., Maccione, A., Tedesco, M., Neukom, S., Koudelka-Hep, M., et al. (2009). Active pixel sensor array for high spatio-temporal resolution electrophysiological recordings from single cell to large scale neuronal networks. Lab Chip 9, 2644-2651. doi: 10.1039/b907394a

Braun, D., and Fromherz, P. (2004). Imaging neuronal seal resistance on silicon chip using fluorescent voltage-sensitive dye. Biophys. J. 87, 1351-1359. doi: 10.1529/biophysj.104.039990

Brown, E. A., Ross, J. D., Blum, R. A., Yoonkey, N., Wheeler, B. C., and Deweerth, S. P. (2008). Stimulus-artifact elimination in a multi-electrode system. IEEE Trans. Biomed. Circuits Syst. 2, 10-21. doi: 10.1109/TBCAS.2008.918285
Buitenweg, J. R., Rutten, W. L., and Marani, E. (2000). Finite element modeling of the neuron-electrode interface. IEEE Eng. Med. Biol. Mag. 19, 46-52. doi: $10.1109 / 51.887245$

Burgard, E. C., and Hablitz, J. J. (1993). Developmental changes in NMDA and non-NMDA receptor-mediated synaptic potentials in rat neocortex. $J$. Neurophysiol. 69, 230-240.

Charvet, G., Rousseau, L., Billoint, O., Gharbi, S., Rostaing, J. P., Joucla, S., et al. (2010). BioMEA: a versatile high-density 3D microelectrode array system using integrated electronics. Biosens. Bioelectron. 25, 1889-1896. doi: 10.1016/j.bios.2010.01.001

Chong, S. A., Benilova, I., Shaban, H., De Strooper, B., Devijver, H., Moechars, D., et al. (2011). Synaptic dysfunction in hippocampus of transgenic mouse models of Alzheimer's disease: a multi-electrode array study. Neurobiol. Dis. 44, 284-291. doi: 10.1016/j.nbd.2011.07.006

Dabrowski, W., Grybos, P., and Litke, A. M. (2004). A low noise multichannel integrated circuit for recording neuronal signals using microelectrode arrays. Biosens. Bioelectron. 19, 749-761. doi: 10.1016/j.bios.2003.08.005

David-Pur, M., Bareket-Keren, L., Beit-Yaakov, G., Raz-Prag, D., and Hanein, Y. (2014). All-carbon-nanotube flexible multi-electrode array for neuronal recording and stimulation. Biomed. Microdevices 16, 43-53. doi: 10.1007/s10544-0139804-6

Demosthenous, A. (2014). Advances in microelectronics for implantable medical devices. Adv. Electron. 2014, 21. doi: 10.1155/2014/981295

Denison, T., Consoer, K., Santa, W., Avestruz, A.-T., Cooley, J., and Kelly, A. (2007). A $2 \mu \mathrm{W} 100 \mathrm{nV} / \mathrm{rtHz}$ chopper-stabilized instrumentation amplifier for chronic measurement of neural field potentials. IEEE J. Solid-State Circuits 42, 2934-2945. doi: 10.1109/JSSC.2007.908664

Egert, U., Schlosshauer, B., Fennrich, S., Nisch, W., Fejtl, M., Knott, T., et al. (1998). A novel organotypic long-term culture of the rat hippocampus on substrateintegrated multielectrode arrays. Brain Res. Brain Res. Protoc. 2, 229-242. doi: 10.1016/S1385-299X(98)00013-0

Enz, C. C., and Temes, G. C. (1996). Circuit techniques for reducing the effects of op-amp imperfections: autozeroing, correlated double sampling, and chopper stabilization. Proc. IEEE 84, 1584-1614. doi: 10.1109/5.542410

Enz, C. C., Vittoz, E. A., and Krummenacher, F. (1987). A Cmos Chopper Amplifier. IEEE J. Solid-State Circuits 22, 335-342. doi: 10.1109/JSSC.1987.1052730

Franks, W., Schenker, I., Schmutz, P., and Hierlemann, A. (2005). Impedance characterization and modeling of electrodes for biomedical applications. IEEE Trans. Biomed. Eng. 52, 1295-1302. doi: 10.1109/TBME.2005.847523

Frey, U., Huang, Y. Y., and Kandel, E. R. (1993). Effects of cAMP simulate a late stage of LTP in hippocampal CA1 neurons. Science 260, 1661-1664. doi: 10.1126/science.8389057

Frey, U., Sedivy, J., Heer, F., Pedron, R., Ballini, M., Mueller, J., et al. (2010). Switchmatrix-based high-density microelectrode array in CMOS technology. IEEE J. Solid-State Circuits 45, 467-482. doi: 10.1109/JSSC.2009.2035196

Gnadt, J. W., Echols, S. D., Yildirim, A., Zhang, H., and Paul, K. (2003). Spectral cancellation of microstimulation artifact for simultaneous neural recording in situ. IEEE Trans. Biomed. Eng. 50, 1129-1135. doi: 10.1109/TBME.2003. 816077

Gosselin, B. (2011). Recent advances in neural recording microsystems. Sensors (Basel) 11, 4572-4597. doi: 10.3390/s110504572

Gosselin, B., Ayoub, A. E., Roy, J. F., Sawan, M., Lepore, F., Chaudhuri, A., et al. (2009). A mixed-signal multichip neural recording interface with bandwidth reduction. IEEE Trans. Biomed. Circuits Syst. 3, 129-141. doi: 10.1109/TBCAS.2009.2013718

Hafizovic, S., Heer, F., Ugniwenko, T., Frey, U., Blau, A., Ziegler, C., et al. (2007). A CMOS-based microelectrode array for interaction with neuronal cultures. J. Neurosci. Methods 164, 93-106. doi: 10.1016/j.jneumeth.2007.04.006

Hampson, R. E., Gerhardt, G. A., Marmarelis, V., Song, D., Opris, I., Santos, L., et al. (2012). Facilitation and restoration of cognitive function in primate prefrontal cortex by a neuroprosthesis that utilizes minicolumn-specific neural firing. $J$. Neural Eng. 9:056012. doi: 10.1088/1741-2560/9/5/056012

Happel, M. F., Jeschke, M., and Ohl, F. W. (2010). Spectral integration in primary auditory cortex attributable to temporally precise convergence of thalamocortical and intracortical input. J. Neurosci. 30, 11114-11127. doi: 10.1523/JNEUROSCI.0689-10.2010

Harrison, R. R., and Charles, C. (2003). A low-power low-noise CMOS amplifier for neural recording applications. IEEE J. Solid-State Circuits 38, 958-965. doi: 10.1109/JSSC.2003.811979 
Heer, F., Hafizovic, S., Franks, W., Blau, A., Ziegler, C., and Hierlemann, A. (2006). CMOS microelectrode array for bidirectional interaction with neuronal networks. IEEE J. Solid-State Circuits 41, 1620-1629. doi: 10.1109/JSSC.2006.873677

Heer, F., Hafizovic, S., Ugniwenko, T., Frey, U., Franks, W., Perriard, E., et al. (2007). Single-chip microelectronic system to interface with living cells. Biosens. Bioelectron. 22, 2546-2553. doi: 10.1016/j.bios.2006.10.003

Hottowy, P., Dabrowski, W., Skoczen, A., and Wiacek, P. (2008). An integrated multichannel waveform generator for large-scale spatio-temporal stimulation of neural tissue. Analog Integr. Circuits Signal Process. 55, 239-248. doi: 10.1007/s10470-007-9125-x

Hottowy, P., Skoczen, A., Gunning, D. E., Kachiguine, S., Mathieson, K., Sher, A., et al. (2012). Properties and application of a multichannel integrated circuit for low-artifact, patterned electrical stimulation of neural tissue. J. Neural Eng. 9:066005. doi: 10.1088/1741-2560/9/6/066005

Hutzler, M., Lambacher, A., Eversmann, B., Jenkner, M., Thewes, R., and Fromherz, P. (2006). High-resolution multitransistor array recording of electrical field potentials in cultured brain slices. J. Neurophysiol. 96, 1638-1645. doi: 10.1152/jn.00347.2006

Jimbo, Y., Kasai, N., Torimitsu, K., Tateno, T., and Robinson, H. P. (2003). A system for MEA-based multisite stimulation. IEEE Trans. Biomed. Eng. 50, 241-248. doi: 10.1109/TBME.2002.805470

Kamioka, H., Maeda, E., Jimbo, Y., Robinson, H. P., and Kawana, A. (1996). Spontaneous periodic synchronized bursting during formation of mature patterns of connections in cortical cultures. Neurosci. Lett. 206, 109-112. doi: 10.1016/S0304-3940(96)12448-4

Kauer, J. A., Malenka, R. C., and Nicoll, R. A. (1988). A persistent postsynaptic modification mediates long-term potentiation in the hippocampus. Neuron 1, 911-917. doi: 10.1016/0896-6273(88)90148-1

Klumperink, E. A. M., Gierkink, S. L. J., van der Wel, A. P., and Nauta, B. (2000). Reducing MOSFET 1/f noise and power consumption by switched biasing. IEEE J. Solid-State Circuits 35, 994-1001. doi: 10.1109/4.848208

Luhmann, H. J., and Prince, D. A. (1991). Postnatal maturation of the GABAergic system in rat neocortex. J. Neurophysiol. 65, 247-263.

Madhavan, R., Chao, Z. C., Wagenaar, D. A., Bakkum, D. J., and Potter, S. M. (2006). Multi-site stimulation quiets network-wide spontaneous bursts and enhances functional plasticity in cultured cortical networks. Conf. Proc. IEEE Eng. Med. Biol. Soc. 1, 1593-1596. doi: 10.1109/IEMBS.2006.260571

Maeda, E., Robinson, H. P., and Kawana, A. (1995). The mechanisms of generation and propagation of synchronized bursting in developing networks of cortical neurons. J. Neurosci. 15, 6834-6845.

Malenka, R. C. (1991). Postsynaptic factors control the duration of synaptic enhancement in area CA1 of the hippocampus. Neuron 6, 53-60. doi: 10.1016/0896-6273(91)90121-F

Masui, Y., Yoshida, T., Sasaki, M., and Iwata, A. (2007). 0.6V Supply complementary metal oxide semiconductor amplifier using noise reduction technique of autozeroing and chopper stabilization. Japn. J. Appl. Phys. 46, 2252-2256. doi: 10.1143/JJAP.46.2252

Meister, M., Pine, J., and Baylor, D. A. (1994). Multi-neuronal signals from the retina: acquisition and analysis. J. Neurosci. Methods 51, 95-106. doi: 10.1016/0165-0270(94)90030-2

Min, Y. J., Kwon, C. K., Kim, H. K., Kim, C., and Kim, S. W. (2012). A CMOS Magnetic hall sensor using a switched biasing amplifier. IEEE Sens. J. 12, 1195-1196. doi: 10.1109/JSEN.2011.2169055

Mohseni, P., and Najafi, K. (2004). Wireless multichannel biopotential recording using an integrated FM telemetry circuit. Conf. Proc. IEEE Eng. Med. Biol. Soc. 6, 4083-4086. doi: 10.1109/IEMBS.2004.1404139

Najafi, K. (1994). Solid-state microsensors for cortical nerve recordings. IEEE Eng. Med. Biol. Mag. 13, 375-387. doi: 10.1109/51.294009

Nemirovsky, Y., Brouk, I., and Jakobson, C. G. (2001). 1/f noise in CMOS transistors for analog applications. IEEE Trans. Electron Devices 48, 921-927. doi: $10.1109 / 16.918240$

Newman, J. P., Zeller-Townson, R., Fong, M. F., Arcot Desai, S., Gross, R. E., and Potter, S. M. (2012). Closed-loop, multichannel experimentation using the open-source neurorighter electrophysiology platform. Front. Neural Circuits 6:98. doi: 10.3389/fncir.2012.00098

Nordhausen, C. T., Maynard, E. M., and Normann, R. A. (1996). Single unit recording capabilities of a 100 microelectrode array. Brain Res. 726, 129-140. doi: 10.1016/0006-8993(96)00321-6
Novak, J. L., and Wheeler, B. C. (1988). Multisite hippocampal slice recording and stimulation using a 32 element microelectrode array. J. Neurosci. Methods 23, 149-159. doi: 10.1016/0165-0270(88)90187-2

Olsson, R. H. 3rd., Buhl, D. L., Sirota, A. M., Buzsaki, G., and Wise, K. D. (2005). Band-tunable and multiplexed integrated circuits for simultaneous recording and stimulation with microelectrode arrays. IEEE Trans. Biomed. Eng. 52, 1303-1311. doi: 10.1109/TBME.2005.847540

Perelman, Y., and Ginosar, R. (2007). An integrated system for multichannel neuronal recording with spike/LFP separation, integrated A/D conversion and threshold detection. IEEE Trans. Biomed. Eng. 54, 130-137. doi: 10.1109/TBME.2006.883732

Roberson, E. D., English, J. D., and Sweatt, J. D. (1996). A biochemist's view of long-term potentiation. Learn. Mem. 3, 1-24. doi: 10.1101/lm.3.1.1

Rodriguez-Perez, A., Delgado-Restituto, M., and Medeiro, F. (2013). A 515 nW, 0-18 dB programmable gain analog-to-digital converter for in-channel neural recording interfaces. IEEE Trans. Biomed. Circuits Syst. 8, 358-370. doi: 10.1109/ TBCAS.2013.2270180

Rolston, J. D., Gross, R. E., and Potter, S. M. (2010). Closed-loop, open-source electrophysiology. Front. Neurosci. 4:31 doi: 10.3389/fnins.2010.00031

Rolston, J. D., Gross, R. E., and Potter, S. M. (2009). NeuroRighter: closed-loop multielectrode stimulation and recording for freely moving animals and cell cultures. Conf. Proc. IEEE Eng. Med. Biol. Soc. 2009, 6489-6492. doi: 10.1109/ IEMBS.2009.5333589

Rousche, P. J., and Normann, R. A. (1992). A method for pneumatically inserting an array of penetrating electrodes into cortical tissue. Ann. Biomed. Eng. 20, 413-422. doi: 10.1007/BF02368133

Rubehn, B., Bosman, C., Oostenveld, R., Fries, P., and Stieglitz, T. (2009). A MEMSbased flexible multichannel ECoG-electrode array. J. Neural Eng. 6:036003. doi: 10.1088/1741-2560/6/3/036003

Schreiner, C. E., and Winer, J. A. (2007). Auditory cortex mapmaking: principles, projections, and plasticity. Neuron 56, 356-365. doi: 10.1016/j.neuron.2007.10.013

Schulz, P. E., and Fitzgibbons, J. C. (1997). Differing mechanisms of expression for short- and long-term potentiation. J. Neurophysiol. 78, 321-334.

Sekirnjak, C., Hottowy, P., Sher, A., Dabrowski, W., Litke, A. M., and Chichilnisky, E. J. (2006). Electrical stimulation of mammalian retinal ganglion cells with multielectrode arrays. J. Neurophysiol. 95, 3311-3327. doi: 10.1152/jn.01168.2005

Sekirnjak, C., Hottowy, P., Sher, A., Dabrowski, W., Litke, A. M., and Chichilnisky, E. J. (2008). High-resolution electrical stimulation of primate retina for epiretinal implant design. J. Neurosci. 28, 4446-4456. doi: 10.1523/JNEUROSCI.513807.2008

Seung Bae, L., Hyung-Min, L., Kiani, M., Uei-Ming, J., and Ghovanloo, M. (2010). An Inductively powered scalable 32-channel wireless neural recording systemon-a-chip for neuroscience applications. IEEE Trans. Biomed. Circuits Syst. 4, 360-371. doi: 10.1109/TBCAS.2010.2078814

Shahrokhi, F., Abdelhalim, K., Serletis, D., Carlen, P. L., and Genov, R. (2010) The 128-channel fully differential digital integrated neural recording and stimulation interface. IEEE Trans. Biomed. Circuits Syst. 4, 149-161. doi: 10.1109/TBCAS.2010.2041350

Sheu, B. J., Scharfetter, D. L., Ko, P. K., and Jeng, M. C. (1987). Bsim-berkeley shortchannel igfet model for Mos-transistors. IEEE J. Solid-State Circuits 22, 558-566. doi: 10.1109/JSSC.1987.1052773

Shimono, K., Brucher, F., Granger, R., Lynch, G., and Taketani, M. (2000). Origins and distribution of cholinergically induced beta rhythms in hippocampal slices. J. Neurosci. 20, 8462-8473.

Shintaku, H., Nakagawa, T., Kitagawa, D., Tanujaya, H., Kawano, S., and Ito, J. (2010). Development of piezoelectric acoustic sensor with frequency selectivity for artificial cochlea. Sens. Actuat. A-Phys. 158, 183-192. doi: 10.1016/j.sna.2009.12.021

Streit, J., Tscherter, A., Heuschkel, M. O., and Renaud, P. (2001). The generation of rhythmic activity in dissociated cultures of rat spinal cord. Eur. J. Neurosci. 14, 191-202. doi: 10.1046/j.0953-816x.2001.01636.x

Sweatt, J. D. (1999). Toward a molecular explanation for long-term potentiation. Learn. Mem. 6, 399-416. doi: 10.1101/lm.6.5.399

Sweatt, J. D. (2009). Mechanisms of Memory, 2nd Edn. London: Academic Press.

Tateno, T., and Jimbo, Y. (1999). Activity-dependent enhancement in the reliability of correlated spike timings in cultured cortical neurons. Biol. Cybern. 80, 45-55. doi: $10.1007 / \mathrm{s} 004220050503$ 
Tateno, T., Jimbo, Y., and Robinson, H. P. (2005). Spatio-temporal cholinergic modulation in cultured networks of rat cortical neurons: evoked activity. Neuroscience 134, 439-448. doi: 10.1016/j.neuroscience.2005.04.055

Tateno, T., Nishikawa, J., Tsuchioka, N., Shintaku, H., and Kawano, S. (2013). A hardware model of the auditory periphery to transduce acoustic signals into neural activity. Front. Neuroeng. 6:12. doi: 10.3389/fneng.2013.00012

Tateno, T., and Robinson, H. P. (2006). Rate coding and spike-time variability in cortical neurons with two types of threshold dynamics. J. Neurophysiol. 95, 2650-2663. doi: 10.1152/jn.00683.2005

Wagenaar, D. A., Pine, J., and Potter, S. M. (2004). Effective parameters for stimulation of dissociated cultures using multi-electrode arrays. J. Neurosci. Methods 138, 27-37. doi: 10.1016/j.jneumeth.2004.03.005

Wagenaar, D. A., and Potter, S. M. (2002). Real-time multi-channel stimulus artifact suppression by local curve fitting. J. Neurosci. Methods 120, 113-120. doi: 10.1016/S0165-0270(02)00149-8

Wagenaar, D. A., and Potter, S. M. (2004). A versatile all-channel stimulator for electrode arrays, with real-time control. J. Neural Eng. 1, 39-45. doi: 10.1088/1741-2560/1/1/006

Watanabe, S., Jimbo, Y., Kamioka, H., Kirino, Y., and Kawana, A. (1996). Development of low magnesium-induced spontaneous synchronized bursting and GABAergic modulation in cultured rat neocortical neurons. Neurosci. Lett. 210, 41-44. doi: 10.1016/0304-3940(96)12653-7

Wattanapanitch, W., Fee, M., and Sarpeshkar, R. (2007). An energy-efficient micropower neural recording amplifier. IEEE Trans. Biomed. Circuits Syst. 1, 136-147. doi: 10.1109/TBCAS.2007.907868

Whitson, J., Kubota, D., Shimono, K., Jia, Y., and Taketani, M. (2006). "Multielectrode arrays: enhancing traditional methods and enable network physiology," in Advances in Network Electrophysiology, eds M. Taketani and M. Baudry (Singapore: Springer), 38-68.

Wichmann, T. (2000). A digital averaging method for removal of stimulus artifacts in neurophysiologic experiments. J. Neurosci. Methods 98, 57-62. doi: 10.1016/S0165-0270(00)00190-4

Winder, D. G., Mansuy, I. M., Osman, M., Moallem, T. M., and Kandel, E. R. (1998). Genetic and pharmacological evidence for a novel, intermediate phase of long-term potentiation suppressed by calcineurin. Cell 92, 25-37. doi: 10.1016/S0092-8674(00)80896-X

Winer, J. A., Miller, L. M., Lee, C. C., and Schreiner, C. E. (2005). Auditory thalamocortical transformation: structure and function. Trends Neurosci. 28, 255-263. doi: 10.1016/j.tins.2005.03.009

Wise, K. D. (1986). The role of thin-films in integrated solid-state sensors. J. Vac. Sci. Technol. A-Vac. Surf. Films 4, 617-622. doi: 10.1116/1.573844

Wise, K. D., Anderson, D. J., Hetke, J. F., Kipke, D. R., and Najafi, K. (2004). Wireless implantable microsystems: high-density electronic interfaces to the nervous system. Proc. IEEE 92, 76-97. doi: 10.1109/JPROC.2003. 820544

Wise, K. D., Angell, J. B., and Starr, A. (1970). An integrated-circuit approach to extracellular microelectrodes. IEEE Trans. Biomed. Eng. 17, 238-247. doi: 10.1109/TBME.1970.4502738

Zou, X., Xu, X., Yao, L., and Lian, Y. (2009). A 1-V 450-nW fully integrated programmable biomedical sensor interface chip. IEEE J. Solid-State Circuits 44, 1067-1077. doi: 10.1109/JSSC.2009.2014707

Conflict of Interest Statement: The authors declare that the research was conducted in the absence of any commercial or financial relationships that could be construed as a potential conflict of interest.

Received: 29 July 2014; accepted: 15 September 2014; published online: 10 October 2014.

Citation: Tateno T and Nishikawa J (2014) A CMOS IC-based multisite measuring system for stimulation and recording in neural preparations in vitro. Front. Neuroeng. 7:39. doi: 10.3389/fneng.2014.00039

This article was submitted to the journal Frontiers in Neuroengineering. Copyright (C) 2014 Tateno and Nishikawa. This is an open-access article distributed under the terms of the Creative Commons Attribution License (CC BY). The use, distribution or reproduction in other forums is permitted, provided the original author(s) or licensor are credited and that the original publication in this journal is cited, in accordance with accepted academic practice. No use, distribution or reproduction is permitted which does not comply with these terms. 